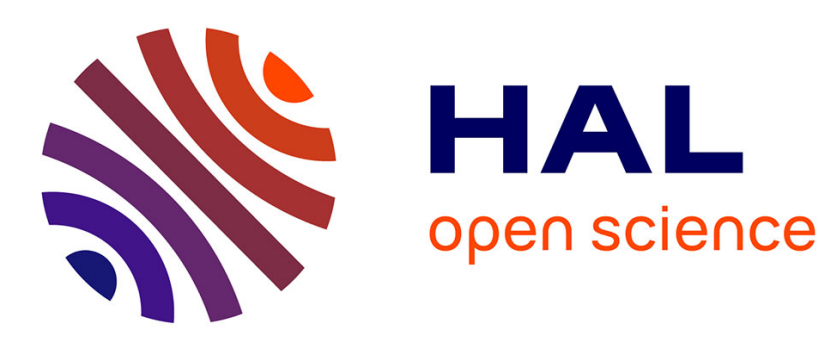

\title{
L'influence de la santé mentale déclarée sur le maintien en emploi
}

Thomas Barnay, Éric Defebvre

\section{To cite this version:}

Thomas Barnay, Éric Defebvre. L'influence de la santé mentale déclarée sur le maintien en emploi. Economie et Statistique / Economics and Statistics, 2016, Travail et santé, 486-487, pp.45-78. hal01343228

\section{HAL Id: hal-01343228 \\ https://hal.science/hal-01343228}

Submitted on 7 Jul 2016

HAL is a multi-disciplinary open access archive for the deposit and dissemination of scientific research documents, whether they are published or not. The documents may come from teaching and research institutions in France or abroad, or from public or private research centers.
L'archive ouverte pluridisciplinaire HAL, est destinée au dépôt et à la diffusion de documents scientifiques de niveau recherche, publiés ou non, émanant des établissements d'enseignement et de recherche français ou étrangers, des laboratoires publics ou privés. 


\title{
L'influence de la santé mentale déclarée sur le maintien en emploi
}

\author{
Thomas Barnay * et Éric Defebvre **
}

\begin{abstract}
Cet article vise à mesurer l'effet causal de la santé mentale déclarée en 2006 (troubles anxieux et épisodes dépressifs) sur le maintien en emploi en 2010. Pour ce faire, nous mobilisons les données de l'enquête Santé et itinéraire professionnel (SIP). De manière à prendre en compte l'endogénéité potentielle inhérente aux variables de santé mentale, nous utilisons une modélisation en Probit bivarié, estimant, d'une part, le maintien en emploi des individus en fonction de leur santé mentale et, d'autre part, la santé mentale, expliquée notamment par des événements marquants de l'enfance. De plus, nous contrôlons nos résultats des caractéristiques socio-économiques, d'emploi, de santé générale, de comportements à risque et d'itinéraire professionnel. Nous montrons que pour les hommes, le fait de déclarer souffrir d'un trouble mental en 2006 est associé à une diminution du maintien en emploi quatre ans plus tard. Concernant les femmes, aucune relation significative de ce type n'est identifiée, après prise en compte de leur santé générale. Les tests de robustesse effectués, notamment sur la période 2007-2010 et sur différentes tranches d'âges, confirment ces résultats.
\end{abstract}

Codes JEL : I14, I18, C35, C36.

Mots clés : santé mentale, emploi, variables instrumentales.

Rappel :

Les jugements et opinions exprimés par les auteurs n'engagent qu'eux mêmes, et non les institutions auxquelles ils appartiennent, ni a fortiori l'Insee.

\footnotetext{
* Université Paris-Est Créteil ; Érudite ; Tepp FR n³435-CNRS et Institut Santé-travail Paris-Est (IST-PE), barnay@u-pec.fr.

${ }^{* *}$ Au moment de la rédaction: Drees, Bureau État de santé de la population (Ministère des Affaires sociales et de la Santé) ; depuis, Érudite, Université Paris-Est Créteil et Tepp FR n`3435-CNRS, eric.defebvre@univ-paris-est.fr.

Les auteurs remercient, pour leurs commentaires sur une première version de cet article, Thibault Brodaty (Érudite, Upec), Roméo Fontaine (Leg, Université de Bourgogne), Aurélia Tison (Université Aix Marseille) et Yann Videau (Érudite, Upec). Les auteurs tiennent aussi à remercier Caroline Berchet (Ined), Marc Collet (Drees), Lucie Gonzalez (Haut Conseil de la Famille), Sandrine Juin (Érudite, Upec et Ined) et Nicolas De Riccardis (Drees) pour avoir répondu à leurs sollicitations ponctuelles sur une version antérieure. Enfin, les auteurs remercient les deux rapporteurs anonymes pour leurs apports sur la version présente. Ils restent évidemment seuls responsables des limites de leur travail.
} 
$\mathbf{L}$ a santé mentale (comprenant les pathologies psychiatriques et les traitements de types médicaments psychotropes sans pathologies psychiatriques identifiées dans les bases de l'assurance maladie) représente $15 \%$ des dépenses de la Cnam-TS en 2011, soit une dépense supérieure à celle du traitement du cancer. Plus de sept millions de personnes sont concernées. Les problèmes de santé mentale sont à l'origine d'une moindre productivité au travail et d'un accroissement du chômage et des arrêts-maladie, ce qui représenterait de 3 à $4 \%$ du PIB selon l'Organisation internationale du travail (2000). Ils expliquent $40 \%$ des incapacités physiques dans les pays à hauts revenus selon l'Organisation mondiale de la santé (2010). De plus, le plan Psychiatrie et santé mentale 2011-2015 atteste de la place majeure de la santé mentale dans les enjeux sociétaux actuels. Il stipule explicitement que l'accès et le maintien dans l'emploi des personnes souffrant de troubles psychiques nécessite un meilleur accompagnement.

La question du maintien dans l'emploi des personnes souffrant de troubles mentaux apparaît essentielle à plusieurs titres. Il est établi qu'une surcharge de travail détériore la santé physique mais aussi mentale (Bell et al., 2012). L'intensité (rythme de travail et manque d'autonomie) et l'insécurité du travail conduisent, en outre, les salariés à faire face à davantage de situations de pénibilité, notamment ressentie. Enfin, le temps partiel, lorsqu'il n'est pas choisi, affecte la santé mentale (Robone et al., 2011).

Les relations entre santé mentale et emploi ont été largement documentées dans la littérature, en particulier pour établir des phénomènes de double causalité. Ces études soulignent en effet la potentielle simultanéité de la relation : alors qu'une santé mentale dégradée influe sur l'emploi, un emploi précaire ou l'exposition à des conditions de travail pénibles peut aussi affecter la santé mentale. Par ailleurs, de façon générale, les indicateurs de santé déclarés se caractérisent par des biais de justification, de mesures et sont soumis à de l'hétérogénéité sociale de déclaration (Shmueli, 2003 ; Étilé et Milcent, 2006 ; Akashi-Ronquest et al., 2011). La santé mentale, lorsqu'elle est subjective (par exemple lorsqu'elle est déclarée par l'individu), semble alors spécifiquement associée à un biais de mesure nécessitant en particulier de démêler les liens entre la santé physique et la santé mentale. À l'instar de la santé physique, des effets de sélection sont également à l'œuvre, une personne souffrant de troubles mentaux se trouvant moins souvent en emploi. La mesure de la santé mentale est aussi potentiellement sujette à un biais spécifique de sélection lié à l'incapacité psychologique de répondre au questionnaire.

Notre objectif est d'établir un effet causal propre de la santé mentale sur le maintien dans l'emploi à partir de données françaises. Cette étude s'inspire de l'étude de Jusot et al. (2008) visant à mesurer l'effet de la santé physique et des comportements à risque sur la sortie de l'emploi quatre ans plus tard. Cependant elle tient compte des biais d'endogénéité liés à la causalité inverse (effet de l'emploi sur la santé mentale). À notre connaissance, aucune étude empirique française n'a mesuré l'effet spécifique de la santé mentale sur le maintien dans l'emploi en corrigeant de ces biais.

Pour ce faire, nous avons recours aux données de l'enquête Santé et itinéraire professionnel $(S I P)$, qui permettent le recueil en 2006 d'une batterie d'indicateurs (socioéconomiques, de santé et de comportements à risque) mais aussi des caractéristiques du parcours professionnel à partir d'un questionnement rétrospectif complet sur les éléments d'emploi et de santé de plus de 13000 individus. La dimension temporelle est autorisée par la seconde vague de 2010 qui réinterroge le panel. L'indicateur de santé mentale repose sur des mesures déclaratives définissant les troubles anxieux généralisés et les épisodes dépressifs caractérisés. À l'aide d'un Probit bivarié, nous évaluons l'effet causal de la santé mentale déclarée en 2006 sur le maintien en emploi en 2010, toutes choses égales par ailleurs et après contrôle de la causalité inverse. L'enjeu est d'identifier un ou des instruments, corrélés à la variable de santé mentale, mais non avec les autres facteurs explicatifs du maintien en emploi ni avec les résidus du modèle.

Nous exposons tout d'abord, dans une revue de la littérature, les principaux résultats empiriques liant santé mentale et statut en emploi. Nous présentons ensuite la base de données et la stratégie empirique retenue. Une dernière section présente les résultats et la discussion conclusive.

\section{Les liens entre santé mentale et emploi}

\section{La mesure de la santé mentale}

La littérature économique établissant le rôle de l'état de santé mentale sur l'emploi retient 
principalement deux définitions de la santé mentale. La première se focalise sur les troubles mentaux lourds, telles les psychoses (Bartel et Taubman, 1986). De nombreuses études évaluent la capacité d'individus atteints de schizophrénie à s'insérer sur le marché du travail (Greve et Herrup Nielsen, 2013). La seconde repose sur des troubles plus répandus mais aussi moins handicapants, comme le stress par exemple. Souvent retenus pour évaluer la santé mentale, ces troubles font l'objet de mesures standardisées et sont présentés sous la forme de scores. Ainsi, le Kessler Psychological Distress Scale (K-10) permet, à partir de 10 questions relatives aux 30 derniers jours passés, d'évaluer l'état mental général de l'individu (Kessler et al., 2003 ; Dahal et Fertig, 2013 ; Zhang et al., 2009). À l'instar du K-10, dans le Short-Form General Health Survey (SF-36), la santé mentale est évaluée, au cours des quatre dernières semaines, à l'aide de réponses à des questions sur le ressenti des individus (enthousiasme, tristesse, manque d'énergie, fatigue,...) (Frijters et al., 2010). Un score comparable a été construit, le Center for Epidemiologic Studies Depression Scale (CES-D), dédié aux travailleurs âgés de 50 à 64 ans, avec des questions plus spécifiques comme l'isolement et l'estime de soi (Chang et Yen, 2011).

Le risque d'agrégation et donc de simplification des scores a cependant justifié la mise en place d'autres indicateurs permettant de mieux approcher le véritable diagnostic de santé mentale. Des indicateurs relatifs aux troubles anxieux généralisés (TAG) et aux épisodes dépressifs caractérisés (EDC) ${ }^{1}$ sont apparus, autorisant une analyse plus approfondie de la santé mentale (Banerjee et al., 2013; Chatterji et al., 2008). Ils permettent d'identifier la population souffrant de ces troubles et de renseigner les symptômes ressentis (cf. annexes 1 et 2). En dépit de leur spécificité et sans qu'ils puissent prétendre se substituer à un diagnostic médical, ces indicateurs s'avèrent robustes pour détecter des troubles mentaux courants (notamment dans le cadre de l'enquête Santé et itinéraire professionnel, comme discuté plus loin).

En outre, le caractère subjectif de la déclaration de la santé en général, et plus particulièrement de la santé mentale, rend difficiles les comparaisons interpersonnelles (Zhang et al., 2009), notamment en raison de biais de déclaration (Shmueli, 2003 ; Devaux et al., 2008). Devaux et al. (op. it.) ont tenté d'évaluer l'importance des biais de déclaration de la santé mentale. Il est ainsi apparu qu'un état de santé latent contribuerait fortement à la santé mentale : deux personnes pourront déclarer des états de santé mentale différents selon leur santé physique. En effet, une personne en mauvais état de santé physique aura davantage tendance à déclarer une santé mentale dégradée qu'une personne en bonne santé physique. Leach et al. (2008) confirment ces résultats et mettent en évidence une forte corrélation entre santé physique et santé mentale, et ce particulièrement chez les femmes.

\section{L'influence de la santé mentale sur l'emploi : une courte revue de la littérature}

\section{Difficultés méthodologiques}

Si la mesure de la santé mentale à partir de données déclaratives n'est pas immédiate, la relation entre la santé mentale et l'emploi est entachée de biais d'endogénéité liés à des phénomènes de causalité inverse et de variables omises ${ }^{2}$. D'un point de vue structurel, on peut concevoir assez facilement que si la santé mentale et l'emploi sont observés simultanément, la relation apparaît comme bidirectionnelle (Banerjee et al., 2013 ; Chatterji et al., 2011). Notamment, l'absence d'emploi peut dégrader la santé mentale des individus (Mossakowski, 2009).

L'omission de variables crée de l'hétérogénéité inobservée, ce qui est aussi potentiellement facteur d'endogénéité dans la mesure de l'effet de l'état de santé mentale sur l'emploi. Les préférences par rapport au risque (Zhang et al., 2009), l'implication de l'individu dans son travail et sa capacité à donner satisfaction (Nelson et Kim, 2008), les traits de personnalité, l'environnement familial (Banerjee et al., 2013), les comportements à risque (tabac, alcool et surcharge pondérale) sont liés tant à la santé mentale qu'à l'emploi. Ces facteurs, qui pour certains restent inobservables dans des enquêtes auprès de ménages, interviennent dès lors comme des facteurs de confusion. Zhang et al. concluent, à partir de données australiennes (données poolées du National Health Survey - NHS) et d'une méthode en Probit multivarié que la consommation de tabac chez les hommes et chez les femmes et la surcharge pondérale chez les femmes augmentent les risques de déclaration

\footnotetext{
1. L'expression "épisode dépressif caractérisé 》 (ou EDC) correspond à la traduction française de l'expression originale issue $d u$ Diagnostic and Statistical Manual version IV (DSM-IV) de " major depressive episode » (MDE), recommandée par la Haute autorité de santé (HAS) en mai 2014.

2. Pour une revue de littérature européenne, cf. Barnay (2015).
} 
de troubles mentaux. Ces comportements ont aussi un effet propre sur la situation sur le marché du travail (Jusot et al., 2008).

Enfin, il est possible de mettre en évidence des biais de justification de la part des individus qui peuvent en effet altérer leurs déclarations d'état de santé, de manière à rationaliser leurs choix sur le marché du travail auprès de l'enquêteur (Zhang et al., 2009). Par exemple, l'absence de participation au marché du travail peut être justifiée ex post par le report d'un plus mauvais état de santé. Lindeboom et Kerkhofs (2009) ont, par exemple, montré, sur données de panel hollandaises et à l'aide de modèles à effets fixes, que les incitations économiques pouvaient être de nature à fausser la déclaration individuelle d'état de santé. Une étude sur un panel irlandais corrobore ces conclusions, après contrôle de l'hétérogénéité inobservée (Gannon, 2009).

\section{Effets nets de la santé mentale sur l'emploi}

Pour répondre à ces difficultés méthodologiques, la littérature empirique recourt aux variables instrumentales et aux techniques de données de panel, ce qui permet de prendre en compte l'hétérogénéité inobservée par l'inclusion d'un effet fixe et la causalité inverse par un décalage temporel entre les variables exogènes et les variables expliquées.

Quelle que soit la mesure de la santé mentale adoptée, les différentes études apparaissent convergentes quant au rôle négatif d'une santé mentale dégradée sur l'emploi. Ainsi, Banerjee et al. (2013) trouvent, à l'aide de modélisations en Probit bivariés et en Two-Stage Least Squares (2SLS) effectuées sur données en coupe, que les personnes déclarant avoir souffert de troubles mentaux (épisodes dépressifs et troubles anxieux) au cours des 12 derniers mois ont beaucoup moins de chance d'être en emploi que les autres au moment de l'enquête ${ }^{3}$. Chatterji et al. (2011) montrent, sur données en coupe et à l'aide de modélisations en deux étapes (Two-Stage Least Squares et Probit bivariés) ainsi que d'une modélisation dite d'Altonji, Elder et Taber (AET, cf. Altonji et al., 2005) prenant en compte l'hétérogénéité inobservée, que ces troubles mentaux, apparus au cours des 12 derniers mois précédant l'enquête, réduisent en moyenne de plus de $15 \%$ les chances d'être en emploi au moment de l'enquête. Une étude américaine, mobilisant des méthodes à variables instrumentales, conclut que la plupart des personnes souffrant de troubles mentaux sont en emploi, mais que des symptômes plus lourds réduisent leur participation au marché du travail (Ojeda et al., 2010). Enfin, des modélisations simultanées sur données poolées taïwanaises confirment qu'une santé mentale dégradée diminue la probabilité de travailler, tout en précisant que la prévalence de ces troubles est moins forte chez les personnes travaillant, induisant ainsi un effet protecteur du travail sur la santé mentale des individus (Chang et al., 2011). Cottini et al. (2013) ont, par ailleurs, confirmé la potentielle causalité inverse, à l'aide d'une méthode en variables instrumentales sur quatre vagues de l'enquête européenne sur les conditions de travail (EWCS) ${ }^{4}$, en soulignant l'effet négatif de mauvaises conditions de travail sur la santé mentale.

Ces effets globaux ne sont pas homogènes selon les groupes d'individus concernés. Ainsi, Zhang etal. (2009) montrent que la santé mentale serait plus pénalisante sur la position sur le marché du travail des travailleurs d'âge moyen (18-49 ans) que des travailleurs seniors (50-64 ans). Les effets de genre sont eux aussi importants. L'effet des troubles mentaux semble plus fort sur l'emploi des hommes que sur celui des femmes ${ }^{5}$ (Ojeda et al., Zhang et al.). Cependant, un tel constat n'est pas consensuel. Ainsi, Frijters et al. (2010) démontrent un effet plus fort de l'état de santé mentale sur l'emploi des femmes que sur celui des hommes, en utilisant des données de panel australiennes (Household, Income and Labour Dynamics in Australia - HILDA) et plusieurs modélisations, incluant un modèle Probit et un modèle à effets fixes à variables instrumentales.

\section{Quel(s) instrument(s) pour la santé mentale?}

Il apparaît nécessaire d'identifier un instrument dont l'influence sur la santé mentale est établie dans la littérature empirique sans qu'il soit corrélé aux autres facteurs explicatifs et aux caractéristiques inobservées.

\footnotetext{
3. Les auteurs ne trouvent pas pour autant un effet significatif de ces troubles sur le nombre de semaines travaillées et de jours d'absence pour les individus en emploi, et cela après contrôle des caractéristiques socioéconomiques standards, du fait de souffrir de maladies chroniques, et de la zone géographique de résidence sur le territoire des États-Unis.

4. European Working Conditions Survey.

5. Chatterii et al. (2011) ne dégagent pas d'effet significatif de la santé mentale sur l'emploi des femmes.
} 


\section{Les déterminants de la santé mentale}

Les déterminants et facteurs associés de la santé mentale sont nombreux dans la littérature et peuvent être classés en trois catégories : les facteurs sociaux, les événements marquants de la vie et les facteurs liés au travail.

Les facteurs sociaux renvoient à la place de l'individu dans la société et aux relations sociales. Plaisier et al. (2008) mettent en évidence trois types de rôles sociaux, protecteurs de la santé mentale: les rôles de partenaire, de parent et de travailleur. Être en couple est associé à une plus forte déclaration de bon état de santé mentale et d'un plus faible risque de dépression et d'anxiété (ibid.; Kelly et al., 2011). Le rôle de parent et celui de partenaire préservent aussi la santé mentale (Plaisier et al.). L'activité professionnelle permet de ralentir le rythme de dépréciation du capital santé mentale, comme le montre une étude sur données de panel tenant compte du caractère endogène des relations entre santé et emploi (Llena-Nozal et al., 2004). Parallèlement, Artazcoz et al. (2004) soulignent que le non-emploi favorise un plus mauvais état de santé mentale chez les hommes, et dans une moindre mesure chez les femmes. Le cumul de ces différents rôles accroit de $39 \%$ la déclaration de bonne santé mentale (Plaisier et al.).

Les événements marquants de la vie jouent aussi un rôle dans la détermination de l'état de santé mentale. Le chômage et $a$ fortiori l'inactivité, en début de vie professionnelle, peuvent favoriser l'apparition de symptômes dépressifs par la suite, comme l'a montré Mossakowski en 2009 sur données longitudinales américaines. En outre, à partir d'un modèle à effets fixes sur données de panel, Lindeboom et al. (2002) établissent que des événements tels que la maladie ou la mort d'un proche ou du partenaire altèrent l'état de santé mentale. De plus, les séparations conjugales et les disputes graves au sein ou à l'extérieur du couple semblent corrélées à un plus mauvais état de santé mentale (Kelly et al., 2010 ; Dalgard et al., 2006). Des difficultés financières passées ou présentes sont aussi souvent associées à l'apparition de troubles mentaux communs, tels les états dépressifs et anxieux (Weich et Lewis, 1998; Laaksonen et al., 2007), tout comme la dégradation de la santé physique (notamment pour les femmes) (Leach et al., 2008). Une santé dégradée ou la présence d'un handicap durant l'enfance a ainsi des conséquences négatives sur la santé mentale à des âges plus avancés, de même que les maladies chroniques, quel que soit l'âge d'apparition (Llena-Nozal et al., 2004).
Des éléments liés au monde du travail peuvent enfin avoir un effet sur l'état de santé mentale. Les contrats de travail atypiques, tels les contrats à temps partiel subis, ont notamment pour effet d'accroître l'occurrence de symptômes dépressifs chez les salariés concernés (Santin et al., 2009). Bildt et Michelsen (2002) montrent, à l'aide de modèles multivariés, que l'exposition à des conditions de travail pénibles peut avoir un effet néfaste sur la santé mentale quatre ans plus tard, avec des différences selon le sexe. Les hommes seraient plus touchés par les changements de tâches et un manque de reconnaissance au travail. Pour les femmes, d'autres conditions spécifiques sont soulignées, telles que le rôle de l'absence de formation et un manque de motivation et de soutien au travail. D'autres facteurs sexués associés à une plus mauvaise santé mentale sont mis en évidence par Cohidon et al. (2010). De manière générale, des facteurs psychosociaux du travail tels l'emprise de l'activité professionnelle sur la vie privée et les contacts avec le public, ainsi que des éléments organisationnels (la répétition de certaines tâches et le manque de coopération dans le travail) sont associés, chez les hommes, à une plus forte déclaration de symptômes dépressifs. Chez les femmes, un début de carrière précoce ou des interruptions involontaires de travail sont aussi corrélés à un plus mauvais état de santé mentale.

\section{Instruments proposés dans la littérature et choix retenus pour cette étude}

Parmi ces nombreux facteurs explicatifs, certains sont mentionnés dans la littérature économique comme des instruments valides et pertinents. Frijters et al. (2010) utilisent le décès d'un ami proche intervenu dans les douze mois précédant l'enquête. Hamilton et al. (1997) recourent aux événements stressants de la vie, à la régularité de la pratique sportive et à la santé mentale retardée, cette dernière étant aussi utilisée par Banerjee et al. (2013). L'état psychologique des parents (Ettner et al., 1997 ; Marcotte et al., 2000), celui des enfants (Ettner et al., 1997 ; Chatterji et al., 2007, 2010), le soutien social (Hamilton et al., 1997 ; French et Alexandre, 2001 ; Ojeda et al., 2009), voire les modifications de législation en matière de santé, de sécurité et de flexibilité du temps de travail (Cottini et Lucifera, 2013) ont aussi été souvent introduits. Ces facteurs ont été privilégiés en raison du rôle joué sur l'état de santé mentale tout en répondant à l'hypothèse d'exogénéité (distance temporelle avec les autres facteurs explicatifs de l'emploi ou absence d'effet direct, 
c'est-à-dire d'effets sur l'emploi non contrôlés par ailleurs dans les modélisations utilisées).

Nous nous inscrivons dans ce cadre en choisissant, comme événements marquants de la vie, des événements durant l'enfance (les violences subies à cette période et le fait d'avoir été élevé par un seul parent) et une variable témoignant de l'état psychologique et de soutien social durant la vie d'adulte (les ruptures conjugales ${ }^{6}$ ), avec une approche différente selon le genre considéré. Nous profitons ainsi d'une distance temporelle certaine (les événements survenus avant 18 ans alors que notre échantillon ne comprend que des individus de 30 ans et plus; les ruptures conjugales antérieures à 2006), et d'une faible probabilité d'effets directs sur l'emploi en 2010, les caractéristiques d'itinéraire professionnel, d'emploi au moment de l'enquête et de comportements à risque en matière de santé étant contrôlés par ailleurs.

\section{Étude empirique}

\section{L'enquête Santé et itinéraire professionnel}

L'enquête Santé et itinéraire professionnel $(S I P)$ donne accès à une description individuelle particulièrement détaillée. Outre les variables socio-économiques classiques (âge, sexe, secteur d'activité, PCS, niveau de diplôme, statut marital), des éléments précis sont recueillis sur l'état de santé physique et mentale (pour plus de précisions, se référer à l'encadré 1).

L'auto-questionnaire renseigne, par ailleurs, les consommations tabagiques actuelles ou passées et la consommation d'alcool décrites de manière précise (fréquence, durée, ...). Les conditions d'emploi (contrat, secteurs, durée du travail, ...) et de travail sont détaillées. La dimension biographique offre enfin la possibilité de réaliser des études diachroniques portant sur les dimensions de santé et de travail depuis la fin des études.

Dans le cadre de notre étude, nous nous intéressons aux personnes ayant répondu à l'enquête SIP en 2006 et en 2010, soit 11016 personnes. Nous sélectionnons les individus âgés de 30 à 55 ans en emploi en 2006, de manière à éviter la prise en compte d'étudiants (cf. encadrés 2 et 3 pour une discussion de la sélection initiale de l'échantillon en 2006 et de l'attrition entre les deux vagues). L'échantillon final est ainsi constitué de 4133 individus, dont 2004 hommes et 2129 femmes.

6. Les variables utilisées par Frijters et al., Ettner et al. ou encore Marcotte et al. ne sont pas disponibles dans l'enquête SIP.

\section{Encadré 1}

\section{L'ENQUÊTE SIP}

Cette étude utilise l'enquête Santé et itinéraire professionnel (SIP). Elle a été réalisée conjointement par la Drees $^{1}$ et la Dares ${ }^{2}$, avec pour objectif de mieux documenter les interactions entre état de santé et parcours professionnel, thèmes jusqu'alors peu abordés simultanément dans les enquêtes françaises. L'enquête retrace ainsi un grand nombre des thèmes importants de la vie des répondants tels que les événements marquants de l'enfance, les études, l'emploi et le travail au cours de toute la carrière, les activités sociales et la santé. Elle compte deux vagues (2006 et 2010), conduites sur le même échantillon représentatif des personnes vivant en ménage ordinaire en France métropolitaine, âgées de 20 à 74 ans, quelle que soit leur situation vis-à-vis de l'emploi (étude, emploi, inactif, retraité...), et avec les mêmes questions. La vague 2010, en plus d'offrir une dimension longitudinale, a bénéficié d'une extension de son volet évaluation des risques psycho-sociaux au travail, à la suite des recommandations du Collège d'expertise sur le suivi statistique des risques psychosociaux au travail. De nouvelles personnes ont par ailleurs été interrogées pour la vague 2010. Deux questionnaires sont proposés à chaque vague : le premier est administré en face-à-face par un enquêteur Insee et renseigne précisément les caractéristiques individuelles et professionnelles ainsi que la santé actuelle des enquêtés. II contient aussi une grille biographique permettant de reconstruire le parcours individuel: l'enfance, les études, la santé, les changements professionnels, les conditions de travail et les événements marquants de la vie. Le second est un auto-questionnaire (rempli par l'enquêté lui-même) ciblant les comportements à risque en matière de santé (poids, consommation de tabac et d'alcool). Au total, 13648 personnes ont répondu en 2006, et 11016 d'entre-elles ont répondu à nouveau en 2010.

\footnotetext{
1. Direction de la Recherche, des Études, de l'Évaluation et des Statistiques - Ministère de la Santé.

2. Direction de l'Animation de la Recherche, des Études et des Statistiques - Ministère du Travail.
} 


\section{Statistiques descriptives}

État de santé de la population en emploi en 2006

Les statistiques descriptives générales sont présentées dans le tableau E de l'annexe 3.
Afin d'appréhender de manière large la santé mentale, nous utilisons les épisodes dépressifs caractérisés (EDC) et les troubles anxieux généralisés (TAG), issus du Mini International Neuropsychatric Interview (MINI), s'appuyant sur le Diagnostic and Statistical manual of

Encadré 2

\section{LA SÉLECTION INITIALE DE L’ÉCHANTILLON EN 2006}

Cette étude ne prétend pas mesurer l'effet de la santé mentale sur l'emploi mais vise uniquement à établir l'effet causal de la santé mentale sur le maintien en emploi, toutes choses égales par ailleurs. Nous écartons de facto la population qui ne travaille pas en 2006 et dont les prévalences déclarées de troubles anxieux et d'épisodes dépressifs sont supérieures à celles de la population en emploi $(22 \%$ vs $6 \%$ chez les hommes; $21 \%$ vs $12 \%$ chez les femmes, cf. annexe 3 , tableaux $A$ et B).

Cette étude ne présente, par conséquent, pas de biais liés à une telle sélection. En revanche, s'il s'agissait de mesurer l'effet de la santé mentale sur la participation au marché du travail, la restriction de l'échantillon aux personnes en emploi en 2006 conduirait a priori à une sous-estimation de l'effet de la santé mentale sur l'emploi. En effet, les caractéristiques socioéconomiques et de santé des personnes souffrant d'un trouble mental en 2006 sont très différentes selon le statut d'emploi. Par exemple, $24 \%$ des personnes déclarant au moins un trouble de santé mentale en 2006 et en emploi à la même date déclarent souffrir de limitations d'activité, contre $52 \%$ chez les personnes sans emploi en 2006.

De plus, une telle étude présenterait des biais méthodologiques importants (simultanéité et causalité inverse en particulier). Une méthode en deux étapes consécutives visant à estimer la probabilité d'être en emploi en 2006 puis la probabilité de se maintenir en emploi conditionnellement à l'équation de participation pourrait alors être menée. Cependant, des problèmes d'identification pourraient apparaître en raison de la difficulté de distinguer rigoureusement les mécanismes explicatifs de la probabilité d'emploi en 2006 de ceux qui déterminent le maintien en emploi entre 2006 à 2010.

Encadré 3

\section{ATTRITION DE L'ÉCHANTILLON INITIAL ENTRE LES VAGUES : PONDÉRATION ET CALAGE}

L'attrition de l'échantillon initial entre les vagues 2006 et 2010 (de 13648 individus à 11 016) peut conduire à sélectionner une population aux caractéristiques spécifiques. Ainsi, à l'origine, des différences sur les taux de réponse à l'enquête 2010 existaient selon l'état de santé perçue, les limitations d'activité, la déclaration d'épisodes dépressifs caractérisés, les gênes motrices et les troubles du sommeil de 2006 (De Riccardis, 2012). Une pondération permettant de tenir compte de cette non-réponse a été mise en place. Elle est calculée à partir de la situation vis-à-vis de l'emploi, des tranches d'unités urbaines, des classes d'âge, des niveaux de diplôme, du sexe et de l'état de santé. Des modèles Logit sont alors utilisés pour estimer les comportements de réponse des enquêtés, selon qu'ils ont effectivement répondu à l'enquête 2010 ou non. Cette procédure permet de repérer des groupes de réponse homogènes $(\mathrm{GRH})$, dans lesquels la probabilité des individus de répondre à l'enquête est équivalente et indépendante entre GRH. Ces GRH sont alors utilisés en tant que stratification de l'échantillon, qui sert de base au tirage d'un second échantilIon avec un taux de sondage égal, pour chaque GRH, à la probabilité de réponse. On peut alors établir des poids, attribués à chaque individu, selon son GRH.
Une fois les données pondérées, il n'existe plus de différences notables concernant les caractéristiques démographiques, socioéconomiques et de santé de notre échantillon entre les non-répondants et les répondants à l'enquête 2010, sur la base de leurs caractéristiques à la première vague (voir annexe 3 , tableaux $C$ et $D$ ).

Le calage permet d'égaliser les totaux pondérés issus de l'échantillon avec ceux connus dans la population générale. II est réalisé sur la moyenne des quatre enquêtes Emploi trimestrielles de l'année 2006 et effectué à l'aide de la macro CalMar (De Riccardis, 2012). Les variables utilisées sont les zones d'études et d'aménagement du territoire, les tranches d'unités urbaines, les classes d'âges, le niveau de diplôme, la nationalité et le nombre d'habitants du logement.

Les procédures de pondération et de calage permettent ainsi de prendre en compte l'attrition entre les deux vagues d'enquête, notamment en raison d'un état de santé général, physique ou mental dégradé, et de faire correspondre l'échantillon de l'enquête à la population générale sur un certain nombre de caractéristiques socio-démographiques. 
Mental disorders (DSM-IV) (cf. encadré 4). Environ $6 \%$ des hommes et $12 \%$ des femmes en emploi déclarent souffrir en 2006 d'au moins un trouble mental (cf. figure I).

De façon générale, les femmes déclarent plus fréquemment des problèmes de santé physique ou mentale. Ainsi, les troubles anxieux (7\%), les épisodes dépressifs ( $8 \%$ ), une santé perçue comme négative $(22 \%)$ et une maladie chronique $(28 \%)$ sont-ils plus largement déclarés par ces dernières que par les hommes (resp. $4 \%, 3 \%, 18 \%$ et $25 \%$ ). Ces comportements de réponse sont fréquemment relevés par la littérature et témoignent, a minima pour une partie d'entre-eux, de biais de déclaration (plutôt à la baisse pour les hommes, et plutôt à la hausse pour les femmes), comme le montrent notamment les études de Devaux et al. (2008) et de Shmueli (2003). A contrario, les comportements à risque en matière de santé sont une caractéristique sensiblement plus masculine. Les fumeurs quotidiens sont plus nombreux chez les hommes $(28 \%$ vs. $24 \%$ chez les femmes), mais c'est surtout pour la consommation à risque d'alcool $(46 \%$ vs. $14 \%)$ et la surcharge pondérale ( $51 \% v s .29 \%)$ que cet écart est le plus élevé.

\section{Troubles de santé et maintien dans l'emploi}

$82 \%$ des hommes en emploi et souffrant d'au moins un trouble mental en 2006 sont encore en

Encadré 4

\section{MESURES ET VALIDITÉ DE LA SANTÉ MENTALE DANS SIP}

Le protocole retenu pour l'enquête SIP s'appuie sur le Diagnostic and Statistical Manual of Mental Disorders (DSM-IV), créé en 1952 par l'Association américaine de psychiatrie (APA). Celui-ci porte exclusivement sur les maladies mentales, à la différence de la Classification internationale des maladies (CIM-10), qui concerne l'ensemble des maladies. Dans SIP, les modules concernant les épisodes dépressifs caractérisés (EDC) et les troubles anxieux généralisés (TAG) du Mini International Neuropsychiatric Interview (MINI) sont repris dans leur intégralité. La construction précise des EDC et des TAG est détaillée dans les annexes 1 et 2. Par le biais de filtres successifs, elle permet de réduire le nombre de "faux positifs ", c'est-à-dire de personnes détectées à tort comme souffrant d'un trouble, au regard des critères diagnostiques.

Selon les critères du DSM-IV évalués par la version du MINI choisie pour SIP, 6,8 \% de la population enquêté a présenté un EDC au cours des deux dernières semaines. Au sein de cette population, $45 \%$ présentent un trouble dépressif récurrent (sur la vie entière). Toujours selon ces critères, 5,7\% de la population a présenté un trouble anxieux généralisé durant les six derniers mois. Comme attendu, ce résultat diffère de ceux issus de l'enquête Santé mentale en population générale (11\% d'épisodes dépressifs au cours des deux dernières semaines et $13 \%$ de TAG). L'algorithme de Santé mentale en population générale (SMPG) s'appuie en effet sur une version Cim-10 (et non DSM-IV) du MINI et détecte plus facilement une dépression ou un TAG.

A contrario, la comparaison avec les résultats de l'enquête Événements de vie et santé (EVS), réalisée sur la même période, également en population générale et avec un protocole identique, fait apparaître des résultats extrêmement proches de ceux de l'enquête SIP (Beck et al., 2010). Dans le Baromètre santé 2005 de l'Inpes (Institut national de prévention et d'éducation pour la santé), utilisé en particulier en épidémiologie, les EDC déclarés par les enquêtés sont aussi mesurés au regard des critères du DSM-IV (précisément à partir du questionnaire Composite International Diagnostic Interview - Short Form, CIDI-SF, recommandé par l'OMS) mais selon une temporalité plus longue (au cours des douze derniers mois). Dans le groupe des 35-54 ans, les prévalences apparaissent donc plus élevées que dans SIP ; $5,4 \%$ chez les hommes et $12,3 \%$ chez les femmes (Beck et Guignard, 2012) (respectivement $4,8 \%$ et $9,7 \%$ pour une population âgée de 30 à 55 ans dans SIP, quel que soit le statut d'activité).

La mesure de la santé mentale dans SIP est donc conforme à une définition plus restrictive (DSM-IV) que celle de la CIM-10, sur des champs similaires (notamment en termes d'âge). Même si le questionnement relatif aux troubles mentaux reprend intégralement la nomenclature proposée par le MINI, il n'a pas valeur de diagnostic. II s'agit plutôt d'entretiens diagnostiques, sur la base de questions posées par un enquêteur Insee, s'appuyant sur l'ensemble des symptômes décrits par le DSM-IV et la CIM-10. II ne doit dès lors pas être considéré comme un diagnostic médical (Bahu et al., 2014). II s'avère cependant que, d'après les résultats d'une post-enquête qualitative réalisée sur certains indicateurs de l'enquête SIP incluant les indicateurs de santé (Guiho-Bailly et al., 2009), les phénomènes de sur-déclaration (fauxpositifs) de troubles mentaux dans l'enquête ne soient pas répandus, alors qu'à l'inverse de la sousdéclaration (faux-négatifs) apparaît. Dans l'étude de l'influence de l'état de santé mentale sur le maintien en emploi, ceci induirait donc une sous-estimation de l'effet de l'état de santé mentale. 
emploi en 2010 , contre $86 \%$ des femmes ${ }^{7}$. Ce sont les troubles anxieux qui ont l'influence la plus forte : $79 \%$ des hommes restent en emploi (vs. $88 \%$ des femmes). Par ailleurs, les indicateurs globaux de santé indiquent des résultats assez similaires entre hommes et femmes. Enfin, concernant les comportements à risque, la consommation quotidienne de tabac ne présente pas d'écart significatif de taux d'emploi entre hommes et femmes. En revanche, la consommation à risque d'alcool $(90 \% v s .93 \%)$ et la surcharge pondérale ( $89 \%$ vs. $93 \%$ ) sont associées à des taux d'emploi plus faible chez les femmes que chez les hommes (cf. figure II).

\section{Santé mentale et état de santé général}

Une forte corrélation entre santé générale et santé mentale est observée dans l'échantillon. Environ $20 \%$ des hommes et des femmes, souffrant d'au moins un trouble mental, déclarent des limitations d'activité, contre $10 \%$ dans l'ensemble de l'échantillon, sans distinction d'état de santé mentale (cf. figure I). Près de $50 \%$ d'entre eux déclarent une mauvaise santé perçue (vs. $20 \%$ en général). Les maladies chroniques ( $45 \%$ vs. $25 \%$ ) et la consommation quotidienne de tabac (30\% vs. $25 \%$ ) sont aussi plus répandues parmi ces personnes. $53 \%$ des hommes et $17 \%$ des femmes souffrant d'un trouble mental déclarent une consommation d'alcool à risque, contre resp. $46 \%$ et $13 \%$ dans l'échantillon global. Enfin, la surcharge pondérale est déclarée à hauteur de $44 \%$ chez les hommes et $31 \%$ chez les femmes ayant un trouble mental, contre resp. $51 \%$ et $29 \%$. Il est intéressant de constater sur ce dernier point que les hommes atteints par un trouble mental sont moins nombreux à déclarer une surcharge pondérale (cf. figure III).

\section{Stratégie économétrique}

La stratégie économétrique repose sur deux étapes permettant de corriger de l'hétérogénéité individuelle puis de l'éventualité d'une causalité inverse.

Dans un premier temps, nous initions une modélisation binômiale de type Probit univarié visant à estimer, parmi les personnes en emploi en 2006, l'effet de la santé mentale en 2006 sur les chances de rester en emploi en 2010 (en emploi $v s$. en non-emploi - variable expliquée $\left.y_{i 2010}\right)$. Plusieurs spécifications sont testées et nous procédons, en outre, à une analyse stratifiée par sexe pour chacune d'entre-elles, en

7. Étant donnée la faiblesse de certains effectifs, il convient cependant d'être prudent quant aux conclusions à tirer de ces statistiques descriptives concernant les troubles mentaux. Les TAG concernent 88 hommes et 195 femmes, les EDC respectivement 91 et 236, et avoir au moins un trouble mental respectivement 150 et 335 .

\section{Figure I}

\section{Prévalence des problèmes de santé, population en emploi en 2006}

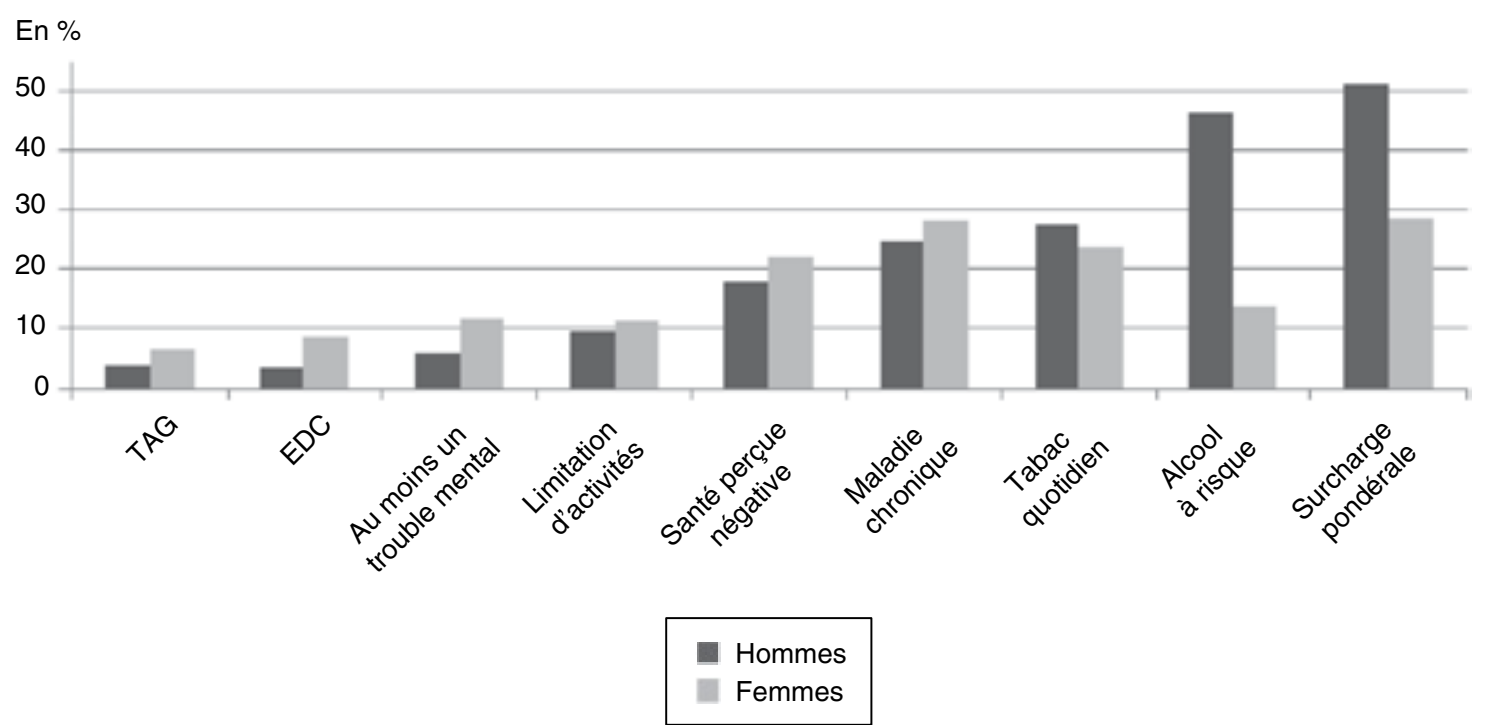

Lecture : statistiques pondérées et calées. $6 \%$ des hommes et $12 \%$ des femmes déclarent souffrir d'au moins un trouble mental (EDC ou TAG) en 2006.

Champ : individus de 30 à 55 ans en emploi en 2006.

Source : enquête Santé et itinéraire professionnel, Insee. 
raison des différences sexuées fortes concernant la santé mentale, soumise notamment à de l'hétérogénéité sociale de déclaration (Leach et al., 2008; Devaux et al., 2008 ; Artazcoz et al., 2004). Nous adoptons une stratégie en trois étapes visant à ajouter progressivement des groupes de variables dans le modèle explicatif. Nous évaluons ainsi la robustesse de la corrélation entre santé mentale en 2006 et emploi en 2010 et identifions progressivement

Figure II

Taux d'emploi en 2010 selon l'état de santé déclaré en 2006, personnes en emploi en 2006

En \%

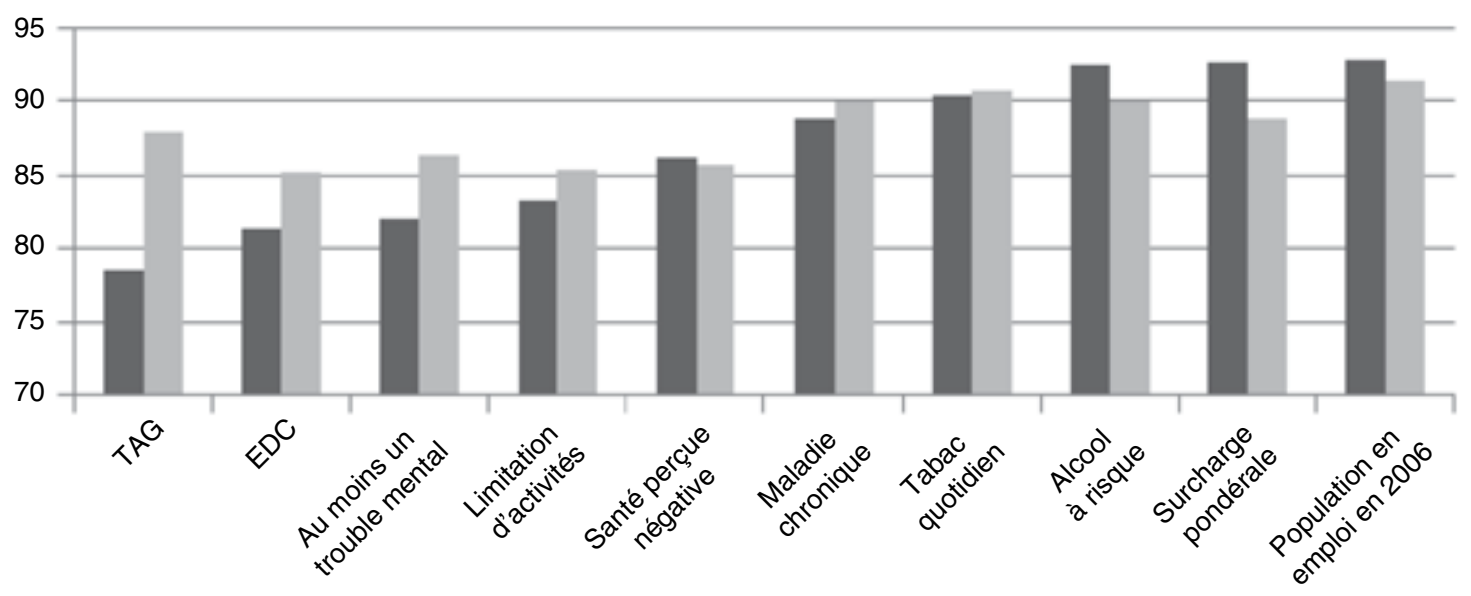

Taux d'emploi en $2010(\mathrm{H})$

Taux d'emploi en 2010 (F)

Lecture : statistiques pondérées et calées. $82 \%$ des hommes en emploi et souffrant d'au moins un trouble mental (TAG ou EDC) en 2006 sont encore en emploi en 2010 , contre $86 \%$ des femmes.

Champ : individus de 30 à 55 ans en emploi en 2006.

Source : enquête Santé et itinéraire professionnel, Insee.

\section{Figure III}

État de santé général des personnes anxieuses et/ou dépressives et en emploi en 2006

En \%

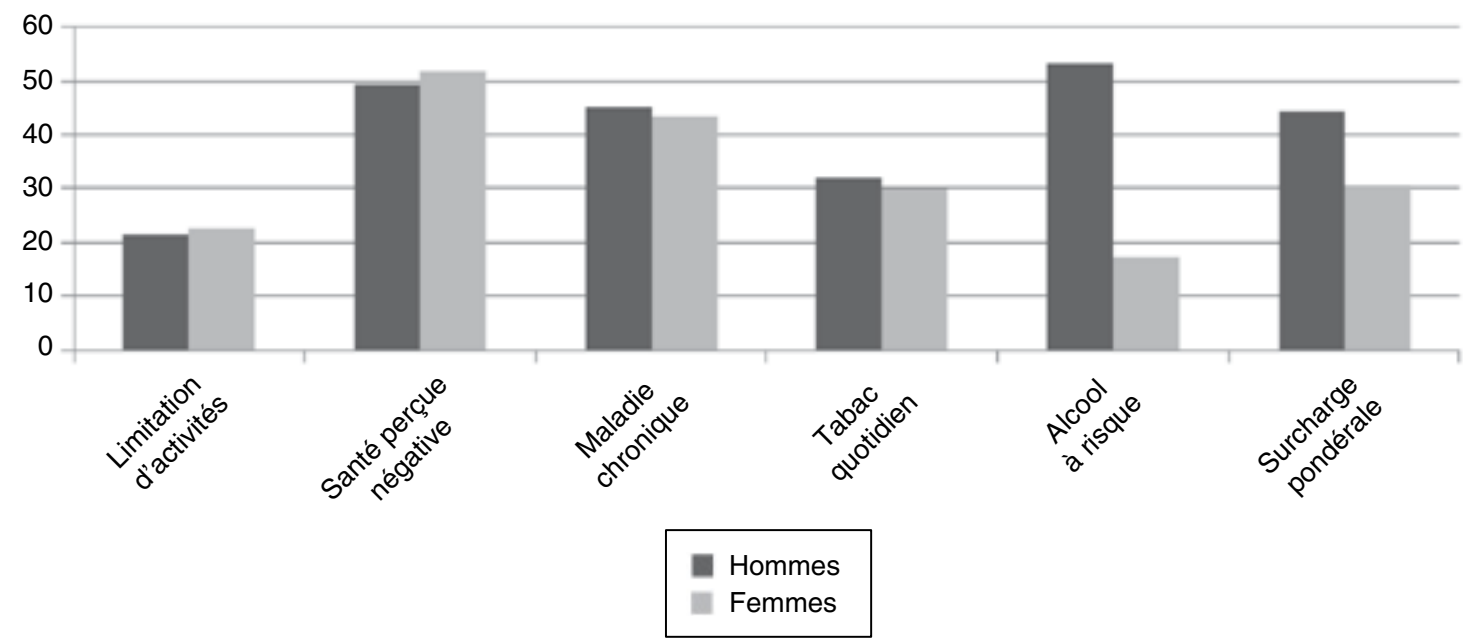

Lecture : statistiques pondérées et calées. $53 \%$ des hommes déclarant des troubles mentaux en 2006 ont aussi une consommation à risque d'alcool en 2006, contre $17 \%$ des femmes.

Champ : individus de 30 à 55 ans en emploi en 2006, ayant déclaré souffrir d'au moins un trouble de santé mentale.

Source : enquête Santé et itinéraire professionnel, Insee. 
des facteurs de confusion dont l'omission pourrait biaiser l'estimation de cette corrélation. La richesse des données de SIP permet de rendre compte de façon exhaustive de l'hétérogénéité individuelle.

La première spécification de base (1) explique le maintien en emploi par la santé mentale, en contrôlant d'un certain nombre de variables socio-économiques standards :

$$
\begin{gathered}
y_{i 2010}=\alpha \text { Ment }_{i 2006}+\beta \text { SocEco }_{i 2006}+\varepsilon_{i} \\
\text { avec Ment } i_{i 2006}=\left\{\begin{array}{c}
1 \text { si }(\text { TAG }=1) \text { ou }(E D C=1) \text { ou }(\text { TAG et EDC }=1) \\
0 \text { sinon }
\end{array}\right.
\end{gathered}
$$

La santé mentale en 2006 (Ment $\left.{ }_{i 2006}\right)$ est ici représentée par une variable binaire simple prenant la valeur 1 lorsque l'individu $i$ souffre d'au moins un trouble mental (soit un trouble anxieux généralisé, soit un épisode dépressif caractérisé, soit des deux à la fois).

Les variables socio-économiques sont représentées par le vecteur $S_{0 c E c o}{ }_{i 2006}$. Elles comprennent l'âge (en tranches quinquennales de 30 à 55 ans), le statut marital, la présence d'enfants, le niveau de diplôme, la PCS, le secteur d'activité, le type d'emploi (public, privé, ou indépendant) et le travail à temps partiel. L'âge joue un rôle majeur sur l'employabilité des individus ainsi que dans la déclaration des troubles mentaux, notamment en raison de biais de déclaration pour certaines tranches (Devaux et al., 2008 ; Schmueli, 2003). Le statut marital et la présence d'enfants dans le ménage peuvent aussi avoir des conséquences sur l'employabilité (notamment pour les femmes) et en termes de santé mentale déclarée, puisque les personnes en couple et ayant des enfants s'avèrent être en meilleure santé (Plaisier et al., 2008 ; Artazcoz et al., 2004). Les caractéristiques liées au travail sont par ailleurs incluses (Llena-Nozal et al., 2004).

Une spécification intermédiaire, (2), est ensuite réalisée, avec l'ajout de trois variables issues du mini-module européen: la santé perçue (prenant la valeur 1 si elle est bonne, et 0 si elle est mauvaise), la prévalence de maladies chroniques et les limitations dans les activités quotidiennes. Ces variables d'état de santé sont présentes de manière à isoler efficacement l'effet propre de la santé mentale sur la position sur le marché du travail (Devaux et al., 2008). Cette modélisation comprend aussi trois variables de comportements à risque : le fait d'être fumeur quotidien, buveur à risque ${ }^{8}$ ou en surpoids. L'insertion de ces variables vise à déterminer dans quelle mesure le rôle de la santé mentale ne transite pas par les comportements à risque (Jusot et al., 2008 ; Butterworth et al., 2011 ; Lim et al., 2000). De tels comportements peuvent avoir des répercussions sur la déclaration de limitations d'activité et en général (Arterburn et al., 2012), sur l'employabilité (Paraponaris et al., 2005), sur l'incidence de maladies et la mortalité prématurée (Teratani et al., 2012) et sur les accidents liés au travail (ibid ; Bourgkard et al., 2008).

Enfin, la troisième et dernière spécification, (3), ajoute deux variables relatives à l'itinéraire professionnel de l'individu obtenues par des informations rétrospectives susceptibles de jouer un rôle sur les caractéristiques individuelles en 2006 et les transitions d'emploi observées entre 2006 et 2010. Il s'agit de contrôler nos résultats d'un parcours professionnel passé instable (phénomène de dépendance d'état), induisant une plus grande fragilité sur le marché du travail (Kelly et al., 2011 ; Mossakowski, 2009). Ces variables concernent le temps passé en emploi long (de plus de 5 ans) et la stabilité du parcours en emploi, représentée par le nombre de transitions effectuées entre emplois de plus de 5 ans, périodes d'emplois courts, périodes de chômage de plus d'un an et enfin périodes d'inactivité.

$$
\begin{aligned}
& y_{i 2010}=\alpha \text { Ment }_{i 2006}+\beta \text { SocEco }_{i 2006}+\delta \text { SantéGen }_{i 2006}+\gamma I P_{i}+\varepsilon_{i} \\
& \text { avec Ment }_{i 2006}=\left\{\begin{array}{c}
1 \operatorname{si}(T A G=1) \text { ou }(E D C=1) \text { ou }(\text { TAG et } E D C=1) \\
0 \text { sinon }
\end{array}\right.
\end{aligned}
$$

Les variables de santé générale et de comportements à risque de 2006 sont présentes dans le vecteur SantéGen $_{i 2006}$ et les variables de contrôle sur l'itinéraire professionnel sont comprises dans le vecteur $I P_{i}$. Ainsi, la relation qui lie le statut en emploi en 2010 et l'état de santé mentale en 2006 est contrôlée de l'état de santé générale, des comportements à risque en matière de santé, et d'éléments en lien avec l'itinéraire professionnel de l'individu.

\footnotetext{
8. Cette notion est issue d'un algorithme de l'AUDIT-C regroupant les consommateurs à risque ponctuel (c'est-à-dire buvant moins de 21 verres par semaine pour les hommes - 14 pour les femmes, et buvant six verres ou plus en une seule occasion moins d'une fois par mois) et à risque chronique (c'est-à-dire buvant 22 verres ou plus par semaine pour les hommes - 15 pour les femmes, et buvant six verres ou plus en une occasion plus d'une fois par mois).
} 
Cependant, comme explicité largement dans la littérature, notre variable de santé mentale souffre potentiellement d'un biais d'endogénéité. La causalité inverse est a priori écartée, dans la mesure où il existe un écart temporel entre notre mesure de la santé mentale (2006) et celle de l'emploi (2010) et du fait que la nature de l'itinéraire professionnel passé (et le statut en emploi en 2006 de facto) est prise en compte (ceci contrôlant des phénomènes de dépendance d'état). Cependant, certaines caractéristiques individuelles (hétérogénéité individuelle inobservée), en lien non seulement avec l'emploi mais aussi avec la santé mentale, ne sont pas prises en compte dans notre modèle, et la mesure de la santé mentale présente de probables biais. Nous sommes donc en présence d'une variable de santé mentale endogène, en raison de la probable hétérogénéité sociale de déclaration, et de variables omises. De manière à prendre en compte cette endogénéité, nous mettons en place une modélisation de type Probit bivarié (cf. encadré 5).

\section{Résultats}

\section{Une mauvaise santé mentale dégrade le maintien dans l'emploi}

Nous testons trois spécifications de la probabilité de rester en emploi en 2010 dans le but de décomposer l'effet de la santé mentale en 2006 mais aussi de tenter de prendre en compte des facteurs de confusion (cf. tableaux 1 et 2).

Le modèle de base (spécification 1) montre, pour les hommes comme pour les femmes, que souffrir d'un TAG et/ou d'un EDC en 2006 conduit à diminuer très significativement la probabilité de rester en emploi en 2010, après contrôle des caractéristiques individuelles et d'emploi de 2006. Les déterminants de l'emploi diffèrent cependant entre hommes et femmes, conformément à ce que d'autres études françaises ont pu observer (Barnay, 2005) et cela, de façon très prononcée. Outre la santé mentale, chez les femmes, les facteurs prédictifs en 2006 d'une sortie de l'emploi quatre ans plus tard sont l'âge (avoir plus de 45 ans), la présence d'enfants, le secteur économique (primaire ou secondaire $v s$. tertiaire) mais aussi le secteur d'activité (privé ou public $v s$. indépendant) et l'exercice d'une activité professionnelle à temps partiel. Il est intéressant de noter qu'au sein de cette population sélectionnée (i.e. en emploi en 2006), le gradient social mesuré à travers la PCS ne joue aucun rôle sur la trajectoire entre 2006 et 2010, toutes choses égales par ailleurs. Chez les hommes, l'exclusion du marché du travail s'accroît avec l'avancée en âge des 50 ans et plus en 2006 mais aussi par l'absence de diplôme, le célibat et la PCS d'appartenance (les ouvriers sont les plus susceptibles de sortir du marché du travail). Les modèles explicatifs entre hommes et femmes ne convergent donc qu'à travers le rôle de la santé mentale et de l'âge.

Dans la spécification 2, on inclut la santé générale (santé perçue, prévalence de maladie et limitations d'activités) et les comportements à risque (consommation quotidienne de tabac, consommation à risque d'alcool et surcharge pondérale). Cette nouvelle spécification permet d'apprécier d'éventuels canaux de transmission indirecte de l'effet de la santé mentale sur l'emploi et de caractériser l'hypothèse d'état de santé latent entre santé mentale et santé physique (Devaux et al., 2008). Dans la population masculine, le coefficient associé à la santé mentale diminue légèrement mais reste très significatif. La limitation d'activité et une consommation régulière de tabac jouent un rôle propre sur la perte d'emploi indépendamment du rôle direct de la santé mentale. Il n'est pas possible de démêler les effets causaux entre santé physique, santé mentale et comportements à risque dans ce type de modèles puisqu'ils sont observés simultanément en 2006, mais la prise en compte explicite de ces variables tend à réduire les inégalités sociales face à l'emploi. Dans la population féminine, le rôle de la santé sur l'emploi ne semble pas transiter par la santé mentale telle que nous la mesurons, mais par la santé perçue et les limitations d'activité. En revanche, les comportements à risque ne semblent présenter aucun caractère discriminant sur le maintien en emploi.

La spécification 3 ajoute à la précédente une information relative à l'itinéraire professionnel passé (en termes de sécurité et de stabilité de l'emploi). Elle permet de contrôler des biais de sélection, la nature de trajectoire professionnelle passée ayant pu influencer à la fois la santé mentale en 2006 mais aussi l'emploi en 2006 et donc en 2010. Même si une trajectoire d'emploi marquée par des emplois longs (donc aussi plus sécurisés) jusque 2006 favorise le maintien dans l'emploi entre 2006 et 2010, l'influence de la santé mentale sur l'emploi des hommes résiste à cette troisième spécification. Chez les femmes, l'itinéraire professionnel n'explique pas la transition d'emploi entre 2006 et 2010 et n'affecte pas le modèle explicatif. 
Encadré 5

\section{PROBIT BIVARIÉ}

Comme suggéré par la littérature (Chatterji et al., 2011 ; Ojeda et al., 2010 ; Frijters et al., 2010) traitant des biais relatifs aux variables de santé mentale, nous mettons en place une méthodologie en Probit bivarié, estimée en maximum de vraisemblance. Elle est équivalente aux méthodes classiques en deux étapes dans un cas linéaire (comme les Two-Stage Least Squares par exemple). Les deux équations à estimer sont les suivantes :

$$
\begin{aligned}
y_{i 2010}= & \alpha \text { Ment }_{i 2006}+\beta S O C E \text { Co }_{i 2006} \\
& + \text { SSantéGen }_{i 2006}+\gamma / P_{i}+\varepsilon_{i}
\end{aligned}
$$

Ment $_{i 2006}=\varphi$ Contr $_{i}+\mu_{i}$

Où le vecteur Contr , contient les mêmes caractéristiques socio-économiques, d'emploi, de santé physique, de comportements à risque et d'itinéraire professionnel de l'individu que celles du modèle (4) et où $\varepsilon_{i}$ et $\mu_{i}$ sont les résidus. Malgré l'inclusion de ces variables de contrôle, il est probable que les résidus de ces deux modèles soient corrélés: $\rho=\operatorname{Corr}\left(\varepsilon_{i}, \mu_{i} \mid\right.$ SocEco $_{i 2006}$, SantéGen $\left._{i 2006}, I P_{i}\right) \neq 0$.

Plusieurs raisons peuvent être avancées. Tout d'abord, dans le cas d'une observation simultanée de ces phénomènes, il existe un fort risque de causalité inverse entre santé mentale et emploi. Dans notre cas, dans la mesure où nous observons deux dates différentes, nous limitons ce risque. II semble cependant possible que la santé mentale en règle générale soit déterminée simultanément avec le statut d'emploi, en raison notamment de phénomènes de dépendance d'état. Ainsi, estimer l'emploi seul reviendrait à omettre une partie du modèle. Enfin, il existe des éléments inobservés tels que les préférences individuelles, les traits de personnalité ou le niveau d'effort, influant non seulement sur l'état de santé mentale mais aussi la capacité de maintien dans l'emploi.

Dans un pareil cas, une modélisation en Probit bivarié est requise en présence de variables qualitatives binaires, ce qui est le cas des variables d'emploi et de santé mentale mobilisées. Elle permet de prendre en compte le caractère endogène d'une variable binaire dont on cherche à évaluer l'effet sur une variable expliquée elle-aussi binaire (Lollivier, 2006). Un tel modèle est estimé en maximum de vraisemblance. Nous mettons donc en place une modélisation supplémentaire, (6), prenant la forme d'un Probit bivarié, utilisant la spécification (3) en guise de modèle principal, et expliquant simultanément l'état de santé mentale par trois variables identifiantes (vecteur /dent $t_{i}$ :

$$
\left\{\begin{array}{c}
y_{i 2010}=\alpha \text { Ment }_{i 2006}+\beta \text { SocEco }_{i 2006}+\delta \text { SantéGen }_{i 2006}+\gamma I P_{i}+\varepsilon_{i} \\
\text { Ment }_{i 2006}=\theta / \text { dent }_{i}+\varphi \text { Contr }_{i}+\mu_{i}
\end{array}\right.
$$

Nous faisons l'hypothèse que les termes d'erreur suivent une loi normale bidimensionnelle :

$$
\left[\begin{array}{l}
\varepsilon_{i} \\
\mu_{i}
\end{array}\right] \rightarrow N\left[\left(\begin{array}{l}
0 \\
0
\end{array}\right),\left(\begin{array}{ll}
1 & \rho \\
\rho & 1
\end{array}\right)\right]
$$

En théorie, il est possible d'estimer un tel modèle sans avoir recours à des variables identifiantes (condition d'exclusion). Néanmoins, dans la littérature empirique, les auteurs préfèrent en général fonder leurs estimations sur la condition d'exclusion et avoir recours aux instruments, procédure jugée plus crédible à condition que les instruments soient forts.

Les variables identifiantes (instruments) retenues dans cette étude ont été choisies en lien avec la littérature sur les déterminants de l'état de santé mentale et sont issues de la partie biographique de SIP : le fait d'avoir été élevé par un seul parent, d'avoir subi des violences durant l'enfance de la part de proches ou à l'école, et enfin le fait d'avoir vécu de nombreuses ruptures conjugales. Nous différencions nos instruments selon le genre ${ }^{1}$ : on retient pour les hommes le fait d'avoir subi des violences et de nombreuses ruptures conjugales et pour les femmes le fait d'avoir subi des violences et d'avoir été élevé par un parent seul.

Utilisant une variable dichotomique endogène d'état de santé mentale, il n'existe pas véritablement de tests spécialisés permettant d'évaluer la pertinence des variables identifiantes. Cependant, dans notre étude, nos instruments ont fait l'objet de tests de corrélation (présentés dans les tableaux A et $B$ de l'annexe 4), de manière à déterminer s'ils répondent aux hypothèses de validité (non-corrélation avec les variables explicatives et les résidus) et de pertinence (corrélation avec l'état de santé mentale). Notamment, dans le cas où les variables identifiantes sont peu pertinentes (peu corrélées avec la santé mentale), ces instruments sont alors dits " faibles ", et sont à l'origine d'une perte de précision des estimations (en raison d'une variance plus élevée de l'estimateur). II est apparu que le fait d'avoir été élevé par un seul parent, d'avoir subi des violences à l'école ou dans l'entourage et enfin d'avoir connu de nombreuses ruptures conjugales permettent de répondre à ces hypothèses. De plus, au regard des estimations de $\rho$, la comparaison des résultats obtenus en Probit bivariés avec ceux obtenus à l'aide des modèles naîfs univariés d'emploi (cf. tableaux 1 et 2) et de santé mentale (cf. annexe 4, tableau C) conforte ces hypothèses (voir infra: "La santé mentale instrumentée »). Néanmoins, ce n'est pas tant le rôle des tests statistiques que celui de la théorie économique de fournir les intuitions nécessaires quant au choix des instruments.

1. Suite à de premières estimations incluant ces trois variables identifiantes pour les hommes et les femmes (non-présentées dans cette version de l'étude), il s'est avéré qu'un usage différencié selon le genre de celles-ci était plus judicieux et permettait une estimation plus précise des modèles. 
De manière à replacer ces résultats dans la littérature empirique, on retrouve la plupart des déterminants classiques influant le marché de l'emploi. Ainsi, l'âge, la présence d'enfants chez les femmes, le niveau de diplôme et la PCS (chez les hommes) et le fait de travailler à temps partiel chez les femmes favorisent le maintien en emploi. Concernant les variables d'état de santé, la santé mentale apparaît très significative chez les hommes, mais pas chez les femmes, ce qui là encore est cohérent avec d'autres travaux (Ojeda et al., 2010; Zhang

Tableau 1

Estimation de la probabilité d'emploi en 2010, population masculine

\begin{tabular}{|c|c|c|c|c|}
\hline & $\begin{array}{l}\text { Probit univarié } \\
\text { (Modèle 1) }\end{array}$ & $\begin{array}{l}\text { Probit univarié } \\
\text { (Modèle 2) }\end{array}$ & $\begin{array}{l}\text { Probit univarié } \\
\text { (Modèle 3) }\end{array}$ & $\begin{array}{l}\text { Probit bivarié } \\
\text { (Résultats V.I.) }\end{array}$ \\
\hline Constante & $1,98^{\star \star \star}$ & $2,16^{\star \star \star}$ & $1,93^{\star \star \star}$ & $1,86^{\star \star \star}$ \\
\hline \multicolumn{5}{|l|}{ Santé mentale en 2006} \\
\hline Au moins un trouble mental & $-0,65^{\star \star \star}$ & $-0,57^{\star \star \star}$ & $-0,56^{\star \star \star}$ & \\
\hline \multicolumn{5}{|l|}{ Santé mentale (instrumentée) en 2006} \\
\hline Au moins un trouble mental & & & & $-0,96^{\star \star}$ \\
\hline \multicolumn{5}{|l|}{ Caractéristiques individuelles en 2006} \\
\hline \multicolumn{5}{|l|}{ Âge (réf. : 30-35 ans) } \\
\hline $35-39$ ans & 0,15 & 0,11 & 0,08 & $-0,04$ \\
\hline $40-44$ ans & $-0,10$ & $-0,23$ & $-0,28$ & $-0,26$ \\
\hline $45-49$ ans & $-0,14$ & $-0,11$ & $-0,19$ & $-0,23$ \\
\hline $50-55$ ans & $-1,01^{\star \star \star}$ & $-1,11^{\star \star \star}$ & $-1,19^{\star \star \star}$ & $-1,26^{\star \star \star}$ \\
\hline En couple (réf. : Seul) & $0,19^{* *}$ & $0,22^{\star *}$ & $0,22^{\star *}$ & 0,15 \\
\hline Présence d'enfants (réf. : Pas d'enfant) & $-0,16$ & $-0,05$ & $-0,06$ & $-0,12$ \\
\hline \multicolumn{5}{|l|}{ Niveau de diplôme (réf. : Bac.) } \\
\hline Pas de diplôme & $-0,42^{\star \star}$ & $-0,38^{\star \star}$ & $-0,36^{*}$ & $-0,45^{\star \star}$ \\
\hline Niveau inférieur au Bac & $-0,20$ & $-0,06$ & $-0,05$ & $-0,09$ \\
\hline Niveau supérieur au Bac & $-0,03$ & $-0,01$ & $-0,01$ & 0,10 \\
\hline \multicolumn{5}{|l|}{ Emploi en 2006} \\
\hline \multicolumn{5}{|l|}{ Sect. d'activité (réf. : Secondaire) } \\
\hline Secteur primaire & $-0,20$ & $-0,18$ & $-0,18$ & $-0,20$ \\
\hline Secteur tertiaire & $-0,02$ & 0,01 & 0,03 & 0,08 \\
\hline \multicolumn{5}{|l|}{ Statut d'activité (réf. : Privé) } \\
\hline Public & $0,21^{*}$ & 0,17 & 0,15 & 0,11 \\
\hline Indépendant & 0,26 & 0,28 & 0,26 & 0,20 \\
\hline \multicolumn{5}{|l|}{ PCS (réf. : Ouvrier) } \\
\hline Agriculteurs exploitants & $1,10^{\star \star \star}$ & $0,94^{\star *}$ & $0,93^{\star *}$ & $0,89^{* *}$ \\
\hline Artisans, ch. d'entr. & $0,53^{\star *}$ & $0,47^{\star}$ & $0,46^{*}$ & $0,75^{\star \star}$ \\
\hline Cadres et prof. intel. sup. & $0,36^{* *}$ & $0,34^{\star *}$ & $0,33^{\star *}$ & $0,32^{*}$ \\
\hline Professions intermédiaires & $0,19^{\star}$ & 0,18 & 0,17 & 0,13 \\
\hline Employés & 0,06 & 0,01 & $-0,01$ & $-0,04$ \\
\hline Temps partiel (réf. : Temps plein) & $-0,33$ & $-0,30$ & $-0,24$ & $-0,09$ \\
\hline \multicolumn{5}{|l|}{ État de santé générale en 2006} \\
\hline Mauvais état de santé perçu & & $-0,13$ & $-0,14$ & $-0,04$ \\
\hline Malade chronique & & 0,02 & 0,01 & 0,01 \\
\hline Limité dans les activités quotidiennes & & $-0,26^{\star}$ & $-0,25^{\star}$ & $-0,30^{\star \star}$ \\
\hline \multicolumn{5}{|l|}{ Comportements à risque en 2006} \\
\hline Fumeur quotidien & & $-0,34^{\star \star \star}$ & $-0,33^{\star \star \star}$ & $-0,37^{\star \star \star}$ \\
\hline Buveur à risque & & $-0,03$ & 0,03 & 0,04 \\
\hline Surcharge pondérale & & 0,05 & 0,05 & 0,07 \\
\hline \multicolumn{5}{|l|}{ Itinéraire professionnel passé } \\
\hline Majorité du parcours en emploi long & & & $0,24^{*}$ & 0,19 \\
\hline Itinéraire professionnel stable & & & 0,04 & 0,02 \\
\hline Rho & & & & $0,22^{\star \star}$ \\
\hline $\mathbf{N}$ & 2004 & 2004 & 2004 & 1860 \\
\hline
\end{tabular}

Lecture : *** : significatif au seuil de $1 \% ;{ }^{* *}$ : significatif au seuil de $5 \%$; : significatif au seuil de $10 \%$.

Champ : hommes en emploi et âgés de 30 à 55 ans en 2006.

Source : enquête Santé et itinéraire professionnel, Insee. 
et al., 2009 et Chatterji et al. 2011). L'étude de Frijters et al. de 2010 va cependant dans le sens contraire, en indiquant un effet plus fort chez les femmes. Ceci peut être expliqué par l'absence de contrôle de l'état de santé générale dans les modélisations proposées dans cette étude, alors que les liens entre santé physique et santé mentale sont forts, notamment chez les femmes (Devaux et al., 2008 ; Leach et al., 2008). À titre d'illustration, nous mettons en exergue un effet significatif de la santé mentale chez les femmes, dans la mesure où nous ne prenons pas

Tableau 2

Estimation de la probabilité d'emploi en 2010, population féminine

\begin{tabular}{|c|c|c|c|c|}
\hline & $\begin{array}{l}\text { Probit univarié } \\
\text { (Modèle 1) }\end{array}$ & $\begin{array}{l}\text { Probit univarié } \\
\text { (Modèle 2) }\end{array}$ & $\begin{array}{l}\text { Probit univarié } \\
\text { (Modèle 3) }\end{array}$ & $\begin{array}{l}\text { Probit bivarié } \\
\text { (Résultats V.I.) }\end{array}$ \\
\hline Constante & $1,60^{\star \star \star}$ & $1,93^{\star \star \star}$ & $1,73^{\star \star \star}$ & $1,73^{\star \star \star}$ \\
\hline \multicolumn{5}{|l|}{ Santé mentale en 2006} \\
\hline Au moins un trouble mental & $-0,33^{\star \star \star}$ & $-0,14$ & $-0,14$ & \\
\hline \multicolumn{5}{|l|}{ Santé mentale (instrumentée) en 2006} \\
\hline Au moins un trouble mental & & & & $-0,17$ \\
\hline \multicolumn{5}{|l|}{ Caractéristiques individuelles en 2006} \\
\hline \multicolumn{5}{|l|}{ Âge (réf. : 30-35 ans) } \\
\hline $35-39$ ans & 0,08 & 0,06 & 0,03 & 0,03 \\
\hline $40-44$ ans & 0,09 & 0,05 & 0,03 & 0,03 \\
\hline $45-49$ ans & $-0,31^{\star *}$ & $-0,25$ & $-0,27$ & $-0,27$ \\
\hline $50-55$ ans & $-0,70^{\star \star \star}$ & $-0,72^{* \star *}$ & $-0,76^{\star \star \star}$ & $-0,76^{\star \star \star}$ \\
\hline En couple (réf. : Seul) & 0,03 & 0,11 & 0,10 & 0,10 \\
\hline Présence d'enfants (réf : Pas d'enfant) & $-0,57^{\star \star \star}$ & $-0,53^{\star \star \star}$ & $-0,52^{\star \star \star}$ & $-0,52^{\star \star \star}$ \\
\hline \multicolumn{5}{|l|}{ Niveau de diplôme (réf. : Bac.) } \\
\hline Pas de diplôme & $-0,21$ & $-0,31$ & $-0,28$ & $-0,28$ \\
\hline Niveau inférieur au Bac & $-0,12$ & $-0,06$ & $-0,05$ & $-0,05$ \\
\hline Niveau supérieur au Bac & 0,03 & $-0,03$ & $-0,04$ & $-0,04$ \\
\hline \multicolumn{5}{|l|}{ Emploi en 2006} \\
\hline \multicolumn{5}{|l|}{ Sect. d'activité (réf. : Secondaire) } \\
\hline Secteur primaire & 0,27 & 0,28 & 0,30 & 0,29 \\
\hline Secteur tertiaire & $0,36^{\star \star \star}$ & $0,43^{\star \star \star}$ & $0,45^{\star \star \star}$ & $0,45^{\star \star \star}$ \\
\hline \multicolumn{5}{|l|}{ Statut d'activité (réf. : Privé) } \\
\hline Public & 0,09 & $0,17^{\star}$ & 0,15 & 0,15 \\
\hline Indépendant & $0,49^{\star \star}$ & $0,45^{\star}$ & $0,48^{\star}$ & $0,47^{\star}$ \\
\hline \multicolumn{5}{|l|}{ PCS (réf. : Ouvrier) } \\
\hline Agriculteurs exploitants & 0,17 & 0,04 & $-0,03$ & $-0,03$ \\
\hline Artisans, ch. d'entr. & $-0,14$ & $-0,21$ & $-0,21$ & $-0,21$ \\
\hline Cadres et prof. intel. sup. & 0,03 & $-0,09$ & $-0,12$ & $-0,12$ \\
\hline Professions intermédiaires & $-0,03$ & $-0,05$ & $-0,07$ & $-0,07$ \\
\hline Employés & 0,10 & 0,02 & 0,01 & 0,01 \\
\hline Temps partiel (réf. : Temps plein) & $-0,19^{\star *}$ & $-0,19^{\star *}$ & $-0,16^{\star}$ & $-0,16^{*}$ \\
\hline \multicolumn{5}{|l|}{ État de santé générale en 2006} \\
\hline Mauvais état de santé perçu & & $-0,26^{\star \star}$ & $-0,25^{\star \star}$ & $-0,24$ \\
\hline Malade chronique & & 0,01 & $-0,01$ & $-0,01$ \\
\hline Limité dans les activités quotidiennes & & $-0,27^{\star \star}$ & $-0,26^{\star \star}$ & $-0,26^{\star}$ \\
\hline \multicolumn{5}{|l|}{ Comportements à risque en 2006} \\
\hline Fumeur quotidien & & $-0,04$ & $-0,01$ & $-0,01$ \\
\hline Buveur à risque & & $-0,11$ & $-0,11$ & $-0,10$ \\
\hline Surcharge pondérale & & $-0,11$ & $-0,11$ & $-0,11$ \\
\hline \multicolumn{5}{|l|}{ Itinéraire professionnel passé } \\
\hline Majorité du parcours en emploi long & & & 0,17 & 0,17 \\
\hline Itinéraire professionnel stable & & & 0,09 & 0,09 \\
\hline Rho & & & & 0,02 \\
\hline $\mathbf{N}$ & 2129 & 2129 & 2129 & 1982 \\
\hline
\end{tabular}

Lecture : *** : significatif au seuil de $1 \% ;{ }^{* *}$ : significatif au seuil de $5 \% ;{ }^{*}$ : significatif au seuil de $10 \%$.

Champ : femmes en emploi et âgées de 30 à 55 ans en 2006.

Source : enquête Santé et itinéraire professionnel, Insee. 
en compte l'état de santé générale (cf. tableau 2 , modèle 1). Un dernier élément concerne les comportements à risque. Le fait d'être fumeur quotidien semble jouer un rôle important sur la position par rapport à l'emploi en 2010 pour les hommes mais pas pour les femmes, ce qui conforte la littérature (Butterworth et al., 2011 ; Jusot et al., 2008). L'alcool et le surpoids ne jouent pas de rôle spécifique sur l'emploi dans nos régressions.

\section{La santé mentale instrumentée}

La dernière colonne des tableaux 1 et 2 présente les résultats du Probit bivarié. Les résultats du modèle de santé mentale (issus du Probit bivarié), sont présentés de manière résumée dans le tableau 3 (pour plus de détails, les résultats complets des estimations des Probit univariés et bivariés de santé mentale sont disponibles dans le tableau C de l'annexe 4) et montrent qu'après avoir contrôlé des caractéristiques individuelles, des variables relatives à l'emploi, à la santé générale, aux comportements à risque et enfin à l'itinéraire professionnel, les trois variables identifiantes (avoir été élevé par un seul parent, avoir subi des violences durant l'enfance et avoir connu de nombreuses ruptures) expliquent une dégradation de l'état de santé mentale alors qu'elles n'expliquent pas de manière propre l'emploi en 2010.

En dépit de la diminution de la précision des estimations du rôle de la santé mentale sur le maintien en emploi, le recours aux instruments permet d'établir une causalité expurgée des biais d'endogénéité inhérents aux facteurs explicatifs utilisés en 2006 et à la relation entre santé mentale et emploi. Le recours à ce type de modélisation semble fondé au regard de la significativité (pour les hommes) du coefficient $\rho$ de corrélation entre les résidus des deux équations simultanées. En outre, l'évolution des résultats entre les modèles d'emploi et de santé mentale indépendants et les modèles bivariés correspondants (cf. tableaux 1, 2 et $\mathrm{C}$ de l'annexe 4) renforce notre analyse. On constate en effet une évolution des coefficients des variables de santé mentale et des variables identifiantes à la hausse (en valeur absolue) chez les hommes, et une stabilité de ceux-ci chez les femmes (ce qui semble correspondre, dans les deux cas, à l'indication donnée par les valeurs respectives de $\rho$ ).

Dans la population masculine, l'effet causal de la santé mentale de 2006 sur l'emploi en 2010 semble donc corroboré par l'analyse en Probit bivarié. Il est aussi possible de réaffirmer le rôle direct de la consommation de tabac sur la perte d'emploi. La santé mentale demeure nondiscriminante sur l'emploi des femmes. Les résultats sont finalement peu modifiés par rapport au modèle 3 du Probit univarié, ce qui semble indiquer que le modèle 3 est bien spécifié et que la causalité chronologique (santé mentale de 2006 - emploi de 2010), qui fonde notre analyse, corrige a priori en partie les biais d'endogénéité. On notera enfin que les résultats obtenus dans nos modèles principaux restent en accord avec la littérature utilisant les mêmes méthodologies.

\section{Tests de robustesse}

Afin de tester la robustesse de nos résultats, nous avons mis en œuvre deux autres spécifications visant à mieux cerner la santé mentale (notamment en différenciant EDC et TAG et en prenant en compte les effets de cumul), à considérer d'autres tranches d'âge ${ }^{9}$ et aussi une temporalité plus courte (il peut être en effet discutable de mesurer l'effet de moyen terme de la santé mentale puisque nous nous projetons, du fait des données, quatre années plus tard).

9. Des tests de sensibilité ont été réalisés, en estimant nos modèles sur les tranches 25-50 ans, 30-50 ans et 25-55 ans. Ces tests, non présentés ici, confirment dans tous les cas nos résultats.

Tableau 3

Estimation de la santé mentale en 2006 (Probit bivarié)

\begin{tabular}{|l|c|c|}
\cline { 2 - 3 } \multicolumn{1}{l|}{} & Hommes & Femmes \\
\hline Instruments & N/A & $0,41^{\star \star \star}$ \\
Élevé par un seul parent & $0,92^{*}$ & $0,47^{\star \star \star}$ \\
A subi des violences durant l'enfance & $0,23^{\star \star}$ & N/A \\
A connu de nombreuses ruptures conjugales & \\
\hline
\end{tabular}

Lecture: ${ }^{* * *}$ : significatif au seuil de $1 \% ;{ }^{* *}$ : significatif au seuil de $5 \% ;{ }^{*}$ : significatif au seuil de $10 \%$. Résultats après contrôle des caractéristiques individuelles, d'emploi, de santé générale et d'itinéraire professionnel passé.

Champ : individus en emploi en 2006 et âgés de 30 à 55 ans.

Source : enquête Santé et itinéraire professionnel, Insee. 


\section{EDC versus TAG}

Nous avons tout d'abord souhaité mieux isoler les effets respectifs des EDC et des TAG sur le maintien en emploi. Le tableau 4 présente, pour les hommes et pour les femmes, les résultats lorsque l'on considère les EDC seuls, les TAG seuls puis un compteur de troubles.

Cette décomposition de la santé mentale ne change pas les résultats dans la population féminine. Même lorsque les femmes déclarent souffrir d'EDC et de TAG, les problèmes de santé mentale n'affectent pas significativement la trajectoire d'emploi. A contrario, elle apparaît intéressante chez les hommes, où l'on remarque en effet que ce sont les TAG qui jouent un rôle négatif sur le maintien dans l'emploi et non les EDC.

\section{Une indicatrice d'emploi sur la période} 2007-2010

La mesure de la santé mentale sur l'emploi est potentiellement sujette à discussion compte tenu de la durée et de la période d'observation. Les trajectoires professionnelles et de santé mentale entre 2006 et 2010 ont pu être significativement affectées par des effets de conjoncture (crise économique de 2009) et de politiques économiques, indépendamment de la santé mentale en 2006. Des politiques de soutien à l'accès ou au maintien en emploi des personnes handicapées ont notamment été mises en place durant cette période. La loi du 11 février 2005 pour l'égalité des droits et des chances, la participation et la citoyenneté des personnes handicapées reconnait, pour la première fois, la dimension psychique du handicap. Le secteur public, tout comme le secteur privé depuis 1987, encourt des sanctions financières en cas de non-respect de l'obligation d'emploi des travailleurs handicapés à hauteur d'au moins $6 \%$ du total des employés. On peut supposer que, sous réserve que la taxe apparaisse assez incitative, cette loi favorise le maintien en emploi des personnes en mauvaise santé mentale souffrant d'un handicap $^{10}$. La forte contraction du marché du travail intervenue en 2009 et la présence résiduelle de dispositifs de cessation précoce d'activité (type dispenses de recherche d'emploi) pour les plus âgés ont pu au contraire favoriser la sortie prématurée du marché du travail des personnes souffrant de problèmes de santé mentale ${ }^{11}$. Il paraît difficile par conséquent de solder ces différents effets sur la période 2006-2010. Il est cependant raisonnable de supposer que les caractéristiques sur le marché du travail ont été significativement différentes avant et après 2009 et probablement hétérogènes entre personnes en bonne et en mauvaise santé mentale.

Pour répondre à cette objection, on considère ainsi le fait d'avoir été au moins 3 ans en emploi entre 2007 et 2010 (et non exclusivement l'emploi en 2010). Les résultats, présentés dans le tableau 5, corroborent l'effet négatif de la déclaration de TAG ou d'EDC sur le maintien en emploi.

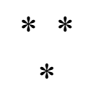

Cette étude démontre qu'un état de santé mentale dégradé diminue directement la capacité des hommes à se maintenir en emploi quatre

10. Cependant Barnay et al. (2016) démontrent que la précédente Loi 1987 en faveur de l'accès à l'emploi des personnes handicapées a globalement été défavorable à ces dernières, les entreprises préférant payer la taxe plutôt que d'embaucher. 11. Sur ce second point, voir Barnay (2008).

\begin{tabular}{|c|c|c|}
\hline \multicolumn{3}{|c|}{$\begin{array}{l}\text { Tableau } 4 \\
\text { Effet de la santé mentale instrumentée en } 2006 \text { sur l'emploi en } 2010 \text { (Probit bivarié), } \\
\text { selon différentes mesures (EDC seul, TAG seul, compteur) }\end{array}$} \\
\hline & Hommes & Femmes \\
\hline \multicolumn{3}{|l|}{ Santé mentale instrumentée } \\
\hline - Souffre d'un EDC & $-0,36$ & $-0,01$ \\
\hline - Souffre d'un TAG & $-0,74^{\star \star}$ & $-0,05$ \\
\hline \multicolumn{3}{|c|}{ Compteur de troubles (réf. : Aucun) } \\
\hline - Un trouble & $-1,10^{\star}$ & $-0,58$ \\
\hline - Deux troubles simultanés & $-1,94^{\star \star \star}$ & $-0,54$ \\
\hline
\end{tabular}

Lecture : ${ }^{* * *}$ : significatif au seuil de $1 \% ;{ }^{* *}$ : significatif au seuil de $5 \% ;{ }^{*}$ : significatif au seuil de $10 \%$. Résultats après contrôle des caractéristiques individuelles, d'emploi, de santé générale et d'itinéraire professionnel passé.

Champ : individus en emploi en 2006 et âgés de 30 à 55 ans.

Source : enquête Santé et itinéraire professionnel, Insee. 
ans plus tard, et cela après contrôle des caractéristiques socio-économiques, d'emploi, de santé générale, de comportements à risque et d'itinéraire professionnel passé. Dans la population féminine, l'état de santé générale reste prédominant pour expliquer leur trajectoire sur le marché du travail. Nos résultats, conformes à ceux de la littérature, apportent des éclairages originaux sur données françaises. La prise en compte dissociée des EDC et des TAG permet d'affirmer que le caractère invalidant de la santé mentale transite par les TAG. De plus, le cumul de troubles mentaux (EDC et TAG) accroît fortement les risques de perte d'emploi. Ces résultats sont corroborés par ailleurs par un travail spécifique sur la période 2007-2010, permettant en partie de s'affranchir des événements intervenus entre 2006 et 2010.

Notre étude confirme le caractère primordial de l'état de santé mentale en termes de maintien en emploi. Il apparaît dès lors pertinent de poursuivre la mise en place de politiques publiques relatives à l'accompagnement des personnes souffrant de troubles mentaux et cela dès l'entrée sur le marché du travail, mais en les étendant aux troubles communs tels que les troubles dépressifs et anxieux, dont la prévalence est élevée en France. Nous apportons de plus de nouveaux éléments par rapport à la différence sexuée de l'influence de l'état de santé mentale, après contrôle de la santé générale. Chez les hommes, les limitations d'activité et les TAG jouent un rôle spécifique et indépendant sur la trajectoire professionnelle. En revanche chez les femmes, ce sont exclusivement les indicateurs de santé générale (santé perçue et limitations d'activité) qui sont en capacité de prédire la situation professionnelle future. Cette différenciation entre les hommes et les femmes est aussi confirmée par l'analyse des déterminants de la santé mentale ${ }^{12}$.

Par conséquent, des mesures d'accompagnement des hommes en situation de souffrance au travail pourraient participer à leur maintien sur le marché du travail. Notamment, le Plan Psychiatrie et santé mentale 2011-2015 affirme l'importance de mesures de prévention du stress au travail, ainsi que des mesures devant permettre de faciliter le maintien et le retour en emploi des personnes souffrant de troubles mentaux, éléments centraux de «la citoyenneté, de l'autonomie et de l'intégration sociale» des individus.

À la suite de cette première étape, plusieurs prolongements peuvent être proposés. Nos résultats démontrent un effet différent de l'état de santé mentale sur le maintien en emploi selon le sexe. Cet écart peut résulter en partie d'une sélection liée à la santé mentale dans l'emploi en 2006 différente selon le sexe $\mathrm{e}^{13}$. Il peut aussi s'expliquer par des différences en termes de normes sociales liées à la perception des troubles mentaux et l'insertion professionnelle, mais aussi par des différences de gravité dans la maladie et des parcours différenciés sur la période 2006-2010 (comme peut en témoigner, concernant les trajectoires de santé, l'état de santé mentale de 2010 des individus malades et en emploi en 2006 - voir tableau G de l'annexe 3). Il serait dès lors intéressant d'établir par quels canaux de transmission transitent ces différences. Le distinguo entre TAG et EDC démontre enfin la sensibilité de nos résultats à la définition de la santé mentale. À ce titre, un score de santé mentale plus détaillé, sous réserve que les échantillons l'autorisent, afin de mieux apprécier la nature et l'intensité de l'altération de la santé, permettrait de mieux juger de son effet sur l'emploi.

12. Différenciation prise en compte dans cette étude à l'aide de variables identifiantes différentes selon le genre.

13. Dans la population masculine souffrant d'au moins un trouble mental en 2006, 68,6\% sont en emploi contre $90,9 \%$ dans la population n'en souffrant pas. Dans la population féminine, ces proportions sont respectivement de $64,5 \%$ et $77,0 \%$ (cf. tableau F de l'annexe 3).

Tableau 5

Estimation de la probabilité d'emploi (indicatrice 2007-2010)

\begin{tabular}{|l|c|c|}
\cline { 2 - 3 } \multicolumn{1}{l|}{} & Hommes & Femmes \\
\hline Santé mentale en 2006 & & \\
A au moins un trouble mental & $-0,46^{\star \star *}$ & $-0,01$ \\
\hline
\end{tabular}

Lecture: ${ }^{* * *}$ : significatif au seuil de $1 \% ;{ }^{* *}$ : significatif au seuil de $5 \% ;{ }^{*}$ : significatif au seuil de $10 \%$. Résultats après contrôle des caractéristiques individuelles, d'emploi, de santé générale et d'itinéraire professionnel passé.

Champ : hommes en emploi en 2006 et âgés de 30 à 55 ans.

Source : enquête Santé et itinéraire professionnel, Insee. 


\section{BIBLIOGRAPHIE}

Akashi-Ronquest N., Carrillo P., Dembling B. et Stern S. (2011), «Measuring the biases in self-reported disability status, evidence from aggregate data », Applied Economics Letters, vol. 18, $\mathrm{n}^{\circ} 11, \mathrm{pp} .1053-1060$.

Altonji J. G., Elder T. E. et Taber C. R. (2005), « Selection on observed and unobserved variables, assessing the effectiveness of Catholic schools », Journal of Political Economy, vol. 113, $\mathrm{n}^{\circ} 1$, pp. 151-184.

Artazcoz L., Benach J., Borrell C. et Cortes I. (2004), «Unemployment and mental health, understanding the interactions among gender, family roles, and social class », American Journal of Public Health, vol. 94, n 1, pp. 82-88.

Arterburn D., Westbrook E., Ludman E., Operskalski B., Linde J., Rohde P., Jeffery R. et Simon G. (2012), « Relationship between Obesity, Depression, and Disability in Middle-Aged Women ", Obesity Research and Clinical Practice,vol. 6, n 3, pp. 197-206.

Bahu M., Mermilliod C., Defebvre É. et De Riccardis N. (2014), «Le choix d'indicateurs de santé, l'exemple de l'enquête SIP 2006 », Document de travail, Série sources et méthodes, ${ }^{\circ} 49$, Drees.

Bahu M., Coutrot T., Mermilliod C. et Rouxel C. (2012), «Appréhender les interactions entre la santé et la vie professionnelle et leur éventuel décalage temporel, un premier bilan d'une enquête innovante, Sip », Document de travail, Série sources et méthodes, $\mathrm{n}^{\circ} 35$, Drees.

Banerjee S., Chatterji P. et Lahiri K. (2013), « Effects of Psychiatric Disorders on Labor Market Outcomes, A Latent Variable Approach Using Multiple Clinical Indicators », CESifo Working Paper, $\mathrm{n}^{\circ}$ 4260, Category 4, Labour Markets.

Barnay T., Duguet E., Le Clainche C. et Videau Y. (2016), «An evaluation of the 1987 French Disabled Workers Act, Better paying than hiring », Document de travail Erudite, $\mathrm{n}^{\circ}$ 01-2016.

Barnay T. (2015), « Health, Work and Working Conditions, A Review of the European Economic Literature », European Journal of Health Economics, DOI, $\mathrm{n}^{\circ} 10.1007 / \mathrm{s} 10198-015-0715-8$, First Online.

Barnay T (2008), « Chômage et invalidité après 50 ans, deux dispositifs alternatifs de sortie de l'emploi pour les seniors en mauvaise santé ? », Économie et Statistique, n 411, pp. 47-63.

Barnay, T. (2005), « Santé déclarée et cessation d'activité », Revue française d'économie, vol. 20, $\mathrm{n}^{\circ} 2$, pp. 73-106.

Bartel A. et Taubman P. (1986), « Some Economic and demographic consequences of Mental Illness "), Journal of Labor Economics, vol. 4, n 2 , pp. 243-256.

Beauregard N., Marchand A. et Blanc M. E. (2011), « What do we know about the non-work determinants of workers' mental health? A systematic review of longitudinal studies », BMC Public Health, ISSN, 1471-2458, vol. 11, pp. 439-454.

Beck F. et Guignard R. (2012), « La dépression en France (2005-2010), prévalence, recours au soin et sentiment d'information de la population », La santé de l'homme, $\mathrm{n}^{\circ} 421$, pp. 43-45.

Beck F., Cavalin C. et Maillochon F. (2010), «Violences et santé en France, état des lieux », Drees, Collection Études et statistiques, Documentation française.

Bell D., Otterbach S. et Sousa-Poza A., (2012), « Work Hours Constraints and Health », Annales d'Economie et Statistique, $\mathrm{n}^{\circ}$ 105-106, pp. 35-54.

Bildt C. et Michelsen H. (2002), « Gender differences in the effects from working conditions on mental health, a 4-year follow-up », International archives of occupational and environmental health, vol. $75, \mathrm{n}^{\circ} 4$, pp. 252-258.

Bourgkard E., Wild P., Massin N. et Meyer J. P. (2008), «Association of physical job demands, smoking and alcohol abuse with subsequent premature mortality, a 9-year follow-up population-based study », Journal of Occupational Health, vol. 50, $\mathrm{n}^{\circ} 1$, pp. 31-40.

Butterworth P., Leach L., Pirkis J. et Kelaher M. (2011), « Poor mental health influences risk and duration of unemployment, a prospective study », Social Psychiatry and Psychiatric Epidemiology, vol. 47, pp. 1013-1021.

Chang H. et Yen S. T. (2011), « Elderly In Taiwan, A Simultaneous Equation Approach », Pacific Economic Review, vol. 16, n 4, pp. 504-519. 
Chatterji P., Alegria M. et Takeuchi D. (2011), «Psychiatric disorders and labor market outcomes, Evidence from the National Comorbidity Survey-Replication », Journal of Health Economics, $\mathrm{n}^{\circ} 30$, pp. 858-868.

Chatterji P., Alegria M. et Takeuchi D. (2008), «Psychiatric disorders and employment, New evidence from the collaborative psychiatric epidemiology survey », NBER Working Paper, $\mathrm{n}^{\circ} 14404$.

Cohidon C., Santin G., Imbernon E. et Goldberg, M. (2010), «Working conditions and depressive symptoms in the 2003 decennial health survey, the role of the occupational category », Social psychiatry and psychiatric epidemiology, vol. 45, $\mathrm{n}^{\circ} 12$, pp. 1135-1147.

Cottini E. et Lucifora C. (2013), « Mental health and working conditions in European countries », Industrial and Labor Relations Review, vol. 66, $\mathrm{n}^{\circ} 4$, pp. 958-988.

Dalgard O., Dowrick C., Lehtinen V., VazquezBarquero J., Casey P., Wilkinson G., AyusoMateos J., Page H. et Dunn G. (2006), « Negative life events, social support and gender difference in depression, A multinational community survey with data from the ODIN study », Social Psychiatry and Psychiatric Epidemiology, pp. 444-451.

Dahal A. et Fertig A. (2013), «An econometric assessment of the effect of mental illness on household spending behavior », Journal of Economic Psychology, n ${ }^{\circ} 37$, pp. 18-33.

DeSimone J. (2002), « Illegal Drug Use and Employment », Journal of Labor Economics, vol. 20, n 4, pp. 952-977.

Devaux M., Jusot F., Sermet C. et Tubeuf S. (2008), «Hétérogénéité sociale de déclaration de l'état de santé et mesure des inégalités de santé », $R F A S, \mathrm{n}^{\circ} 1$, pp. 29-47.

Étilé F. et Milcent C. (2006), «Income-related reporting heterogeneity in self-assessed health, evidence from France $»$, Health economics, vol. $15, n^{\circ} 9$, pp. 965-981.

Ettner S. L., Frank R. G. et Kessler R. C. (1997), «The impact of psychiatric disorders on labor market outcomes », NBER Working Paper Series, $\mathrm{n}^{\circ} 5989$.

French M. T. et Alexandre P. K. (2001), « Labor supply of poor residents in metropolitan Miami,
Florida, The role of depression and the co-morbid effects of substance use », The Journal of Mental Health Policy and Economics, vol. 4, $\mathrm{n}^{\circ} 4$, pp. 161-173.

Frijters P., Johnston D. W. et Shields M. A. (2010), « Mental Health and Labour Market Participation, Evidence from IV Panel Data Models », IZA DP, $\mathrm{n}^{\circ} 4883$.

Gannon B., (2009), « The Influence of Economic Incentives on Reported Disability Status », Health Economics, 18(7), pp. 743-759.

Greve J. et Herrup Nielsen L. (2013), « Useful beautiful minds-An analysis of the relationship between schizophrenia and employment ", Journal of Health Economics, vol. 32, $\mathrm{n}^{\circ} 6$, pp. 1066-1076.

Guiho-Bailly M.-P., Bertin C., Dubre J.Y., Lancien N., Machefer J. et Parent D. (2009), « Rapport subjectif au travail, sens des trajets professionnels et construction de la santé - Rapport final, Tome 1 », Document de travail, Série Études et Recherches, $\mathrm{n}^{\circ}$ 95, Drees.

Hamilton V. H., Merrigan P. et Dufresne E. (1997), «Down and Out, Estimating the Relationship between Mental Health and Unemployment », Health Economics; n 6, pp. 397-406.

Johnson J. V., Hall E. M. et Theorell T. (1989), «Combined effects of job strain and social isolation on cardiovascular disease morbidity and mortality in a random sample of the Swedish male working population », Scandinavian journal of work, environment \& health, vol. $15, \mathrm{n}^{\circ} 4$, pp. 271-279.

Jusot F., Khlat M., Rochereau T. et Sermet C. (2008), « Job loss from poor health, smoking and obesity, a national prospective survey in France », Journal of Epidemiology \& Community Health, $\mathrm{n}^{\circ} 62$, pp. 332-337.

Karasek R. A. Jr. (1979), « Job demands, job decision latitude, and mental strain, Implications for job redesign », Administrative science quarterly, vol. $24, \mathrm{n}^{\circ} 2$, pp. 285-308.

Kelly B., Lewin T., Stain H., Coleman C., Fitzgerald M., Perkins D., Carr V., Fragar L., Fuller J., Lyle D. et Beard J. (2011), « Determinants of mental health and well-being within rural and remote communities », Social psychiatry and psychiatric epidemiology, vol. 46, $\mathrm{n}^{\circ} 12$, pp. 1331-1342. 
Kessler R., Greenberg P., Mickelson K., Meneades L. et Wang P. (2001), « The effects of chronic medical conditions on work loss and work cutback », Journal of Occupational and Environmental Medicine (JOEM), n 43,pp. 218-225.

Laaksonen M., Martikainen P., Rahkonen O. et Lahelma E. (2008), « Explanations for gender differences in sickness absence, evidence from middle-aged municipal employees from Finland », Occupational and Environmental Medicine, vol. $65, \mathrm{n}^{\circ} 5$, pp. 325-330.

Leach L., Christensen H., Mackinnon A., Windsor T. et Butterworth P. (2008), « Gender differences in depression and anxiety across the adult lifespan, the role of psychosocial mediators », Social psychiatry and psychiatric epidemiology, vol. 43, n 12, pp. 983-998.

Lim D., Sanderson K. et Andrews G. (2000), «Lost Productivity Among Full-Time Workers with Mental Disorders », The Journal of Mental Health Policy and Economics, vol. 3, $\mathrm{n}^{\circ} 3$, pp. 139-146.

Lindeboom M. et Kerkhofs M. (2009), « Health and work of the elderly, Subjective health measures, reporting errors and endogeneity in the relationship between health and work », Journal of Applied Econometrics, vol. 24, $\mathrm{n}^{\circ}$ 6, pp. 1024-1046.

Lindeboom M., Portrait F. et van den Berg G. (2002), «An Econometric Analysis of the Mental-Health Effects of Major Events in the Life of Older Individuals », Health Economics, vol. 11, $\mathrm{n}^{\circ} 6$, pp. 505-520.

Llena-Nozal A., Lindeboom M. et Portrait F. (2004), " The effect of work on mental health, does occupation matter? ", Health Economics, vol. $13, \mathrm{n}^{\circ} 10$, pp. 1045-1062.

Lollivier S. (2006), Économétrie avancée des variables qualitatives, Economica, collection Économie et Statistiques Avancées.

Lu C., Frank R. G., Liu Y. et Shen J. (2009), «The Impact of Mental Health on Labour Market Outcomes in China », The Journal of Mental Health Policy and Economics, $\mathrm{n}^{\circ} 12$, pp. 157-166.

Marcotte D. E., Wilxox-Gok V. et Redmond D. P. (2000), «The labor market effects of mental illness, the case of affective disorders », dans Salkever D. et Sorkin A. (éditeurs), The Economics of Disability. JAI Press, Greenwich, pp. 181-210.
Mossakowski K. (2009), « The Influence of Past Unemployment Duration on Symptoms of Depression among Young Women and Men in the United States » American Journal of Public Health, vol. $99, \mathrm{n}^{\circ} 10$, pp. 1826-1832.

Nelson R., Kim J. (2008), « Survival of the fittest, Impact of mental illness on employment duration », Pharmacotherapy outcomes, Research Center, University of Utah.

Ojeda V. D., Frank R. G., McGuire T. G. et Gilmer T. P. (2010), « Mental Illness, Nativity, Gender And Labor Supply », Health Economics, $\mathrm{n}^{\circ} 19$, pp. 396-421.

Paraponaris A., Saliba B. et Ventelou B., (2005), «Obesity, weight status and employability, Empirical evidence from a French national survey », Economics \& Human Biology, Elsevier, vol. 3, $\mathrm{n}^{\circ} 2$, pp. 241-258.

Plaisier I., De Bruijn J. G., Smit J. H., De Graaf R., Ten Have M., Beekman A. T., Van Dyck R. et Penninx B. W. (2008), « Work and family roles and the association with depressive and anxiety disorders, differences between men and women », Journal of Affective Disorders, vol. 105, n¹-3, pp. 63-72.

Renna F. (2008), «Alcohol Abuse, Alcoholism, And Labor Market Outcomes, Looking For The Missing Link », Industrial and Labor Relations Review, vol. 62, $\mathrm{n}^{\circ}$ 1, pp. 92-103.

Riccardis de N. (2012), "Traitements de la non-réponse et calages pour l'enquête santé et itinéraire professionnel de $2010 »$, Document de travail, Série sources et méthodes, ${ }^{\circ} 36$, Drees.

Robone S., Jones A. et Rice N. (2011), « Contractual conditions, working conditions and their impact on health and well-being ", European Journal of Health Economics, vol. 12, n 5, pp. 429-444.

Santin G., Cohidon C., Goldberg M. et Imbernon E. (2009), « Depressive Symptoms and Atypical Jobs in France from the 2003 Decennial Health Survey ", American journal of industrial medicine 2009, vol. 52, n 10, pp. 799-810.

Shmueli A. (2003), « Socio-economic and demographic variation in health and in its measures, the issue of reporting heterogeneity ", Social Science and Medicine, $\mathrm{n}^{\circ}$ 57, pp. 125-134.

Teratani T., Morimoto H. et Sakata K. (2012), « Dose-response relationship between tobacco or 
alcohol consumption and the development of diabetes mellitus in Japanese male workers », Drug and Alcohol Dependence, vol. 125, pp. 276-282.

Weich S. et Lewis G. (1998), « Material standard of living, social class, and the prevalence of the common mental disorders in Great Britain »,
Journal of Epidemiology and Community Health, $\mathrm{n}^{\circ} 52$, pp. 8-14.

Zhang X., Zhao X. et Harris A. (2009), « Chronic diseases and labour force participation in Australia », Journal of Health Economics, $\mathrm{n}^{\circ} 28$, pp. 91-108. 


\section{LES ÉPISODES DÉPRESSIFS CARACTÉRISÉS (EDC) DANS L'ENQUÊTE SIP}

Les EDC sont répertoriés en deux temps. Deux questions faisant usage de filtres sont d'abord posées :

- «Au cours des deux dernières semaines, vous êtes-vous senti(e) particulièrement triste, cafardeux(se), déprimé(e), la plupart du temps au cours de la journée, et ce, presque tous les jours? Oui / non "

- «Au cours des deux dernières semaines, aviez-vous presque tout le temps le sentiment de n'avoir plus goût à rien, d'avoir perdu l'intérêt ou le plaisir pour les choses qui vous plaisent habituellement ? Oui / non "

Ensuite, si une des deux questions filtres reçoit une réponse positive, une troisième question est alors posée, de manière à connaître les symptômes précis : "Au cours de ces deux dernières semaines, lorsque vous vous sentiez déprimé(e) et/ou sans intérêt pour la plupart des choses, avez-vous vécu l'une des situations suivantes ? Cocher dès que la réponse est " oui ", plusieurs réponses positives possibles. "

- Votre appétit a notablement changé, ou vous avez pris du poids ou perdu du poids sans en avoir l'intention (variation au cours du mois de $+/-5 \%$ ).

- Vous aviez des problèmes de sommeil presque toutes les nuits (endormissement, réveils nocturnes ou précoces, dormir trop).
- Vous parliez ou vous vous déplaciez plus lentement que d'habitude, ou au contraire vous vous sentiez agité(e), et vous aviez du mal à rester en place, presque tous les jours.

- Vous vous sentiez presque tout le temps fatigué(e), sans énergie, et ce presque tous les jours.

- Vous vous sentiez sans valeur ou coupable, et ce presque tous les jours.

- Vous aviez du mal à vous concentrer ou à prendre des décisions, et ce presque tous les jours.

- Vous avez eu à plusieurs reprises des idées noires (comme penser qu'il vaudrait mieux que vous soyez mort(e)), ou vous avez pensé à vous faire du mal.

À l'aide des réponses fournies, deux algorithmes sont alors mis en place, en accord avec les critères du Diagnostic and Statistical Manual (DSM-IV). Un individu souffre d'EDC si :

- une réponse positive aux deux questions filtre et quatre symptômes sont répertoriés ;

- deux réponses positives aux deux questions filtre et trois symptômes sont répertoriés. 


\section{LES TROUBLES ANXIEUX GÉNÉRALISÉS (TAG) DANS L'ENQUÊTE SIP}

Les TAG sont repérés en utilisant un système similaire de questions filtre.

Trois questions sont posées.

- «Au cours des six derniers mois, avez-vous eu l'impression de vous faire trop de souci à propos de tout et de rien, vous êtes-vous senti(e) excessivement préoccupé(e), inquiet(inquiète), anxieux(se) pour des problèmes de la vie de tous les jours, au travail/à l'école, à la maison ou à propos de votre entourage ? Oui / Non "

En cas de réponse positive :

- «Avez-vous ce type de préoccupations presque tous les jours? Oui / Non »

En cas de réponse positive :

- «Vous est-il difficile de contrôler ces préoccupations ou vous empêchent-elles de vous concentrer sur ce que vous avez à faire ? Oui / Non "

Dans un second temps et dans la mesure où l'enquêté répond positivement aux trois questions filtre, une autre question est alors posée, de manière à connaître les symptômes précis : "Au cours des six derniers mois, lorsque vous vous sentiez particulièrement préoccupé(e), inquiet (inquiète), anxieux (se), vous arrivait-il souvent : "

- De vous sentir agité(e), tendu(e), les nerfs à fleur de peau?

- D'avoir les muscles tendus?

- De vous sentir fatigué(e), faible ou facilement épuisé(e) ?

- D’avoir des difficultés à vous concentrer ou des passages à vide?

- D'être particulièrement irritable?

- D'avoir des problèmes de sommeil (difficultés d'endormissement, réveils au milieu de la nuit, réveils précoces ou dormir trop)?

Pour qu'une personne souffre d'un trouble anxieux généralisé, elle doit répondre positivement aux trois questions filtre, puis à trois des six symptômes décrits par la suite. Ce protocole est conforme à celui utilisé par le DSM-IV. 


\section{STATISTIQUES DESCRIPTIVES}

Tableau A

Analyse de la sélection - Population en emploi vs. sans emploi en 2006

En \%

\begin{tabular}{|c|c|c|c|c|}
\hline & \multirow{2}{*}{\multicolumn{2}{|c|}{ Hommes }} & & \\
\hline & & & \multicolumn{2}{|c|}{ Femmes } \\
\hline & $\begin{array}{l}\text { Population en } \\
\text { emploi en } 2006\end{array}$ & $\begin{array}{l}\text { Population sans } \\
\text { emploi en } 2006\end{array}$ & $\begin{array}{l}\text { Population en } \\
\text { emploi en } 2006\end{array}$ & $\begin{array}{l}\text { Population sans } \\
\text { emploi en } 2006\end{array}$ \\
\hline \multicolumn{5}{|l|}{ Santé mentale, 2006} \\
\hline Au moins un trouble mental & 5,9 & 22,2 & 11,6 & 21,0 \\
\hline Pas de trouble mental & 94,1 & 77,8 & 88,4 & 79,0 \\
\hline EDC & 3,4 & 16,7 & 8,3 & 16,4 \\
\hline Pas d'EDC & 96,6 & 83,3 & 91,7 & 83,6 \\
\hline TAG & 3,5 & 13,2 & 6,6 & 13,1 \\
\hline Ne souffre pas de TAG & 96,5 & 86,8 & 93,4 & 86,9 \\
\hline \multicolumn{5}{|l|}{ Caractéristiques individuelles, 2006} \\
\hline $30-34$ ans & 17,3 & 11,6 & 16,0 & 15,9 \\
\hline $35-39$ ans & 21,7 & 10,9 & 20,2 & 15,1 \\
\hline $40-44$ ans & 20,2 & 16,4 & 19,9 & 16,4 \\
\hline $45-49$ ans & 20,1 & 19,6 & 21,4 & 18,5 \\
\hline $50-55$ ans & 20,8 & 41,5 & 22,5 & 34,1 \\
\hline En couple & 82,1 & 55,0 & 77,6 & 71,5 \\
\hline Pas en couple & 17,9 & 45,0 & 22,4 & 28,5 \\
\hline Au moins un enfant & 12,2 & 5,1 & 8,3 & 6,1 \\
\hline Pas d'enfant & 87,8 & 94,9 & 91,7 & 93,9 \\
\hline Sans diplôme & 8,0 & 15,1 & 6,7 & 15,3 \\
\hline Diplôme inférieur au Baccalauréat & 45,8 & 53,6 & 39,1 & 45,8 \\
\hline Baccalauréat & 18,2 & 14,2 & 19,1 & 17,2 \\
\hline Diplôme supérieur au Baccalauréat & 26,3 & 16,1 & 33,3 & 18,5 \\
\hline \multicolumn{5}{|l|}{ Caractéristiques d'emploi, 2006} \\
\hline Secteur primaire & 9,0 & & 3,1 & \\
\hline Secteur secondaire & 21,0 & & 9,1 & \\
\hline Secteur tertiaire & 70,0 & & 87,7 & \\
\hline Privé & 66,7 & & 58,9 & \\
\hline Public & 19,1 & & 29,1 & \\
\hline Indépendant & 10,9 & & 6,6 & \\
\hline Agriculteurs exploitants & 4,7 & & 1,2 & \\
\hline Artisans, commerçants, chefs d'entreprise & 7,0 & & 4,3 & \\
\hline Cadres et professions intellectuelles sup. & 16,4 & & 11,1 & \\
\hline Professions Intermédiaires & 24,1 & & 22,2 & \\
\hline Employés & 12,7 & & 45,1 & \\
\hline Ouvriers & 29,8 & & 9,2 & \\
\hline Travail à temps partiel & 3,0 & & 30,7 & \\
\hline Travail à temps plein & 97,0 & & 69,3 & \\
\hline \multicolumn{5}{|l|}{ Santé générale, 2006} \\
\hline Bonne santé perçue & 82,1 & 48,9 & 77,8 & 61,2 \\
\hline Mauvaise santé perçue & 17,9 & 51,1 & 22,2 & 38,8 \\
\hline Pas de maladie chronique & 75,3 & 56,6 & 71,9 & 60,3 \\
\hline Maladie chronique & 24,7 & 43,4 & 28,1 & 39,7 \\
\hline Pas de limitation d'activités & 90,7 & 59,8 & 88,5 & 75,1 \\
\hline Est limité dans ses activités & 9,3 & 40,2 & 11,5 & 24,9 \\
\hline
\end{tabular}


Tableau A (suite)

\begin{tabular}{|c|c|c|c|c|}
\hline & \multicolumn{2}{|c|}{ Hommes } & \multicolumn{2}{|c|}{ Femmes } \\
\hline & $\begin{array}{l}\text { Population en } \\
\text { emploi en } 2006\end{array}$ & $\begin{array}{l}\text { Population sans } \\
\text { emploi en } 2006\end{array}$ & $\begin{array}{l}\text { Population en } \\
\text { emploi en } 2006\end{array}$ & $\begin{array}{l}\text { Population sans } \\
\text { emploi en } 2006\end{array}$ \\
\hline \multicolumn{5}{|l|}{ Comportements à risque, 2006} \\
\hline Fumeur quotidien & 27,5 & 47,8 & 23,6 & 24,5 \\
\hline Pas fumeur quotidien & 72,5 & 52,2 & 76,4 & 75,5 \\
\hline Buveur à risque & 46,2 & 42,2 & 13,6 & 13,1 \\
\hline Pas buveur à risque & 53,8 & 57,8 & 86,4 & 86,9 \\
\hline Surcharge pondérale & 51,3 & 46,7 & 28,5 & 41,6 \\
\hline Poids normal ou sous poids & 48,7 & 53,3 & 71,5 & 58,4 \\
\hline \multicolumn{5}{|l|}{ Itinéraire professionnel passé } \\
\hline Majorité du parcours en emploi long & 83,5 & 45,3 & 71,7 & 58,0 \\
\hline Majorité du parcours hors d'emploi long & 16,5 & 54,7 & 28,3 & 42,0 \\
\hline Itinéraire professionnel stable & 74,3 & 51,5 & 68,9 & 27,0 \\
\hline Itinéraire professionnel instable & 25,7 & 48,6 & 31,1 & 73,0 \\
\hline
\end{tabular}

Lecture : statistiques pondérées et calées. 5,9\% des hommes en emploi en 2006 souffrent d'au moins un trouble mental à la même date, contre $22,2 \%$ chez ceux sans emploi.

Champ : personnes en emploi et en non-emploi et âgées de 30 à 55 ans en 2006

Source : enquête Santé et itinéraire professionnel, Insee. 
Tableau B

Analyse de la sélection - caractéristiques principales des individus déclarant au moins un trouble mental en 2006, selon leur statut d'emploi de 2006

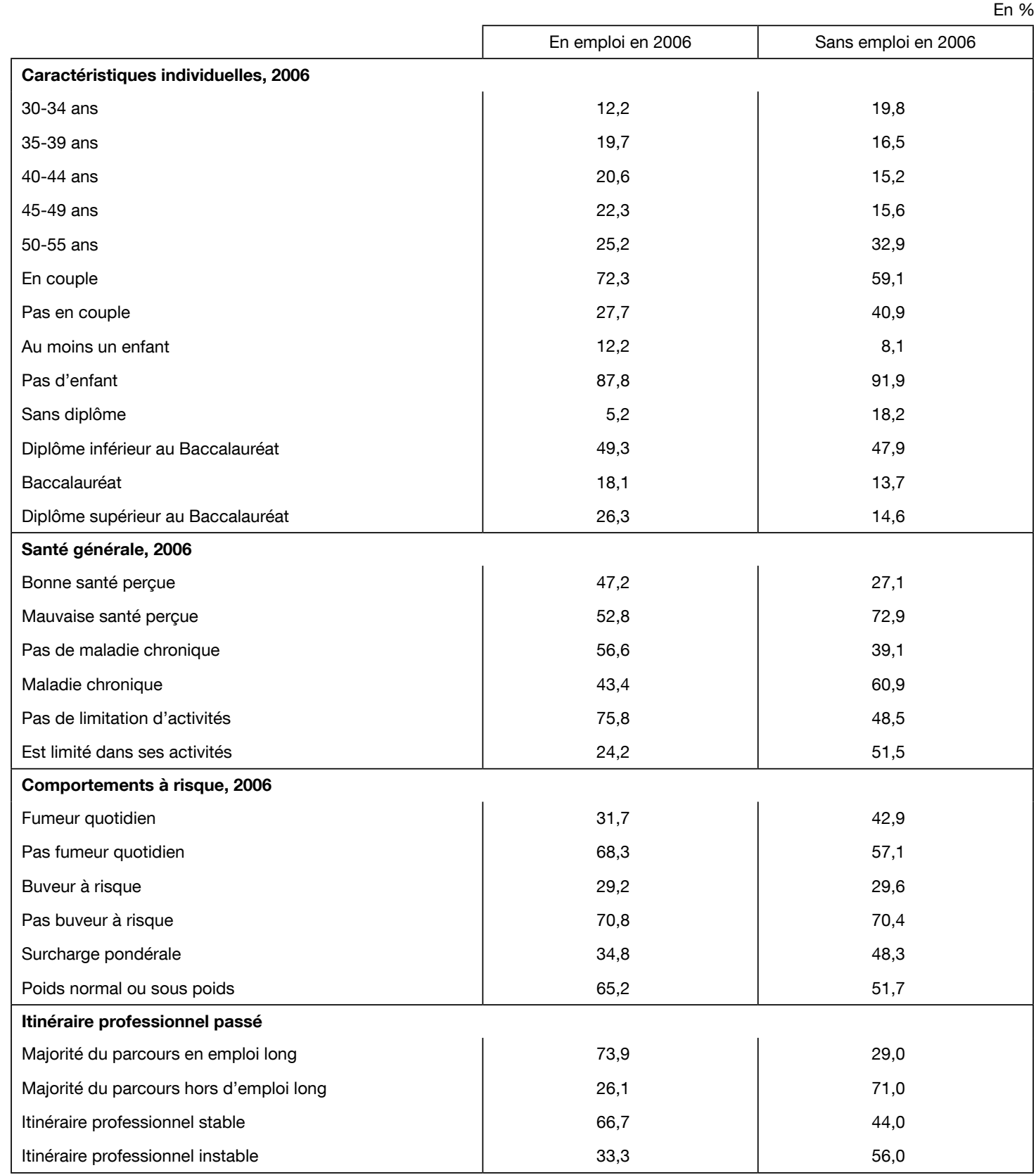

Lecture : statistiques pondérées et calées. $24,2 \%$ des personnes déclarant au moins un trouble de santé mentale en 2006 et en emploi à la même date déclarent souffrir de limitations d'activité, contre 51,5\% chez les personnes sans emploi en 2006.

Champ : personnes âgées de 30 à 55 ans en 2006 et déclarant au moins un trouble de santé mentale en 2006.

Source : enquête Santé et itinéraire professionnel, Insee. 
Tableau C

Analyse de l'attrition - Population en panel (interrogée en 2006 et 2010)

vs. population de l'attrition (interrogée en 2006 et pas en 2010)

En $\%$

\begin{tabular}{|c|c|c|c|c|}
\hline & \multicolumn{2}{|c|}{ Hommes } & \multicolumn{2}{|c|}{ Femmes } \\
\hline & $\begin{array}{l}\text { Population } \\
\text { en panel }\end{array}$ & $\begin{array}{l}\text { Population } \\
\text { de l'attrition }\end{array}$ & $\begin{array}{l}\text { Population } \\
\text { en panel }\end{array}$ & $\begin{array}{l}\text { Population } \\
\text { de l'attrition }\end{array}$ \\
\hline \multicolumn{5}{|l|}{ Santé mentale, 2006} \\
\hline Au moins un trouble mental & 5,9 & 5,9 & 11,6 & 13,5 \\
\hline Pas de trouble mental & 94,1 & 94,1 & 88,4 & 86,5 \\
\hline EDC & 3,4 & 4,4 & 8,3 & 9,0 \\
\hline Pas d'EDC & 96,6 & 95,2 & 91,7 & 91,0 \\
\hline TAG & 3,5 & 3,7 & 6,6 & 6,9 \\
\hline Ne souffre pas de TAG & 96,5 & 96,3 & 93,4 & 93,1 \\
\hline \multicolumn{5}{|l|}{ Caractéristiques individuelles, 2006} \\
\hline $30-34$ ans & 17,3 & 18,9 & 16,0 & 15,3 \\
\hline $35-39$ ans & 21,7 & 21,5 & 20,2 & 23,5 \\
\hline $40-44$ ans & 20,2 & 21,3 & 19,9 & 21,6 \\
\hline $45-49$ ans & 20,1 & 17,8 & 21,4 & 18,6 \\
\hline $50-55$ ans & 20,8 & 20,5 & 22,5 & 21,0 \\
\hline En couple & 82,1 & 71,7 & 77,6 & 61,8 \\
\hline Pas en couple & 17,9 & 28,3 & 22,4 & 38,2 \\
\hline Au moins un enfant & 12,2 & 23,8 & 8,3 & 18,4 \\
\hline Pas d'enfant & 87,8 & 86,2 & 91,7 & 81,6 \\
\hline Sans diplôme & 8,0 & 8,0 & 6,7 & 7,8 \\
\hline Diplôme inférieur au Baccalauréat & 45,8 & 46,7 & 39,1 & 40,4 \\
\hline Baccalauréat & 18,2 & 14,8 & 19,1 & 21,0 \\
\hline Diplôme supérieur au Baccalauréat & 26,3 & 29,1 & 33,3 & 29,4 \\
\hline \multicolumn{5}{|l|}{ Caractéristiques d'emploi, 2006} \\
\hline Secteur primaire & 9,0 & 4,8 & 3,1 & 3,5 \\
\hline Secteur secondaire & 21,0 & 16,6 & 9,1 & 8,2 \\
\hline Secteur tertiaire & 70,0 & 78,6 & 87,7 & 88,3 \\
\hline Privé & 66,7 & 65,2 & 58,9 & 60,2 \\
\hline Public & 19,1 & 20,7 & 29,1 & 28,4 \\
\hline Indépendant & 10,9 & 10,0 & 6,6 & 5,9 \\
\hline Agriculteurs exploitants & 4,7 & 1,4 & 1,2 & 1,2 \\
\hline Artisans, commerçants, chefs d'entreprise & 7,0 & 9,6 & 4,3 & 4,3 \\
\hline Cadres et professions intellectuelles sup. & 16,4 & 16,8 & 11,1 & 12,0 \\
\hline Professions Intermédiaires & 24,1 & 20,7 & 22,2 & 22,9 \\
\hline Employés & 12,7 & 12,9 & 45,1 & 44,7 \\
\hline Ouvriers & 29,8 & 32,4 & 9,2 & 8,0 \\
\hline Travail à temps partiel & 3,0 & 4,1 & 30,7 & 25,1 \\
\hline Travail à temps plein & 97,0 & 95,9 & 69,3 & 75,0 \\
\hline \multicolumn{5}{|l|}{ Santé générale, 2006} \\
\hline Bonne santé perçue & 82,1 & 79,7 & 77,8 & 74,7 \\
\hline Mauvaise santé perçue & 17,9 & 20,3 & 22,2 & 25,3 \\
\hline Pas de maladie chronique & 75,3 & 79,0 & 71,9 & 73,5 \\
\hline Maladie chronique & 24,7 & 21,1 & 28,1 & 26,5 \\
\hline Pas de limitation d'activités & 9,3 & 88,5 & 88,5 & 88,2 \\
\hline Est limité dans ses activités & 90,7 & 11,5 & 11,5 & 11,8 \\
\hline
\end{tabular}


Tableau C (suite)

\begin{tabular}{|c|c|c|c|c|}
\hline & \multicolumn{2}{|c|}{ Hommes } & \multicolumn{2}{|c|}{ Femmes } \\
\hline & $\begin{array}{l}\text { Population } \\
\text { en panel }\end{array}$ & $\begin{array}{l}\text { Population } \\
\text { de l'attrition }\end{array}$ & $\begin{array}{l}\text { Population } \\
\text { en panel }\end{array}$ & $\begin{array}{l}\text { Population } \\
\text { de l'attrition }\end{array}$ \\
\hline \multicolumn{5}{|l|}{ Comportements à risque, 2006} \\
\hline Fumeur quotidien & 27,5 & 34,9 & 23,6 & 30,1 \\
\hline Pas fumeur quotidien & 72,5 & 65,1 & 76,4 & 69,9 \\
\hline Buveur à risque & 46,2 & 44,0 & 13,6 & 14,1 \\
\hline Pas buveur à risque & 53,8 & 36,0 & 86,4 & 85,9 \\
\hline Surcharge pondérale & 51,3 & 48,6 & 28,5 & 21,3 \\
\hline Poids normal ou sous poids & 48,7 & 51,4 & 71,5 & 78,7 \\
\hline \multicolumn{5}{|l|}{ Itinéraire professionnel passé } \\
\hline Majorité du parcours en emploi long & 83,5 & 69,9 & 71,7 & 69,4 \\
\hline Majorité du parcours hors d'emploi long & 16,5 & 30,1 & 28,3 & 30,6 \\
\hline Itinéraire professionnel stable & 74,3 & 76,0 & 68,9 & 67,6 \\
\hline Itinéraire professionnel instable & 25,7 & 24,0 & 31,1 & 32,5 \\
\hline
\end{tabular}

Lecture : statistiques pondérées et calées. Chez les hommes interrogée en 2006 et en 2010 (panel), 3,4 \% d'entre-eux déclarent un EDC contre 4,4\% chez ceux uniquement interrogés en 2006 (attrition).

Champ : personnes en emploi et en non-emploi et âgées de 30 à 55 ans en 2006.

Source : enquête Santé et itinéraire professionnel, Insee. 
Tableau D

Analyse de l'attrition - Appartenance à l'attrition/au panel selon la santé mentale et le statut en emploi en 2006

\begin{tabular}{|c|c|c|}
\hline & Attrition & Panel \\
\hline \multicolumn{3}{|l|}{ Santé mentale, 2006} \\
\hline Au moins un trouble mental & 18,6 & 81,4 \\
\hline Pas de trouble mental & 16,9 & 83,1 \\
\hline EDC & 19,5 & 80,5 \\
\hline Pas d'EDC & 16,9 & 83,1 \\
\hline TAG & 17,7 & 82,3 \\
\hline Ne souffre pas de TAG & 17,0 & 83,0 \\
\hline \multicolumn{3}{|l|}{ Statut en emploi en 2006} \\
\hline En emploi & 16,0 & 84,0 \\
\hline Sans emploi & 22,1 & 77,9 \\
\hline \multicolumn{3}{|l|}{ Croisements santé et emploi, 2006} \\
\hline \multicolumn{3}{|l|}{ En emploi en 2006} \\
\hline Au moins un trouble mental & 16,6 & 83,4 \\
\hline Pas de trouble mental & 15,9 & 84,1 \\
\hline EDC & 17,1 & 82,9 \\
\hline Pas d'EDC & 15,9 & 84,1 \\
\hline TAG & 15,7 & 84,3 \\
\hline Ne souffre pas de TAG & 16,0 & 84,0 \\
\hline \multicolumn{3}{|l|}{ Pas en emploi en 2006} \\
\hline Au moins un trouble mental & 22,6 & 77,4 \\
\hline Pas de trouble mental & 22,0 & 78,0 \\
\hline EDC & 23,5 & 78,1 \\
\hline Pas d'EDC & 21,9 & 76,5 \\
\hline TAG & 21,6 & 78,4 \\
\hline Ne souffre pas de TAG & 22,2 & 77,8 \\
\hline
\end{tabular}

Lecture : statistiques pondérées et calées. Parmi les individus déclarant en 2006 souffrir d'au moins un trouble mental, 18,6 \% n'ont pas été réinterrogés en 2010 et 81,4 \% l'ont été. Chez les individus n'ayant pas déclaré de troubles mentaux en 2006, 16,9 \% n'ont pas été réinterrogés.

Champ : hommes en emploi et âgées de 30 à 55 ans en 2006.

Source : enquête Santé et itinéraire professionnel, Insee. 
Tableau E

Statistiques descriptives générales

\begin{tabular}{|c|c|c|c|c|}
\hline & \multicolumn{2}{|c|}{ Hommes ( \%) } & \multicolumn{2}{|c|}{ Femmes ( \%) } \\
\hline & Proportion & $\begin{array}{c}\text { Probabilité } \\
\text { d'être en emploi } \\
\text { en } 2010\end{array}$ & Proportion & $\begin{array}{c}\text { Probabilité } \\
\text { d'être en emploi } \\
\text { en } 2010\end{array}$ \\
\hline \multicolumn{5}{|l|}{ Santé mentale, 2006} \\
\hline Au moins un trouble mental & 5,9 & 82,0 & 11,6 & 86,3 \\
\hline Pas de trouble mental & 94,1 & 93,1 & 88,4 & 92,0 \\
\hline EDC & 3,4 & 81,3 & 8,3 & 85,1 \\
\hline Pas d'EDC & 96,6 & 92,8 & 91,7 & 91,9 \\
\hline TAG & 3,5 & 78,5 & 6,6 & 87,8 \\
\hline Ne souffre pas de TAG & 96,5 & 93,0 & 93,4 & 91,6 \\
\hline \multicolumn{5}{|l|}{ Caractéristiques individuelles, 2006} \\
\hline $30-34$ ans & 17,3 & 96,3 & 16,0 & 92,9 \\
\hline $35-39$ ans & 21,7 & 96,6 & 20,2 & 93,7 \\
\hline $40-44$ ans & 20,2 & 95,2 & 19,9 & 96,2 \\
\hline $45-49$ ans & 20,1 & 94,8 & 21,4 & 91,1 \\
\hline $50-55$ ans & 20,8 & 79,8 & 22,5 & 83,9 \\
\hline En couple & 82,1 & 93,1 & 77,6 & 91,5 \\
\hline Pas en couple & 17,9 & 89,3 & 22,4 & 90,6 \\
\hline Au moins un enfant & 12,2 & 96,3 & 8,3 & 85,6 \\
\hline Pas d'enfant & 87,8 & 91,9 & 91,7 & 91,9 \\
\hline Sans diplôme & 8,0 & 86,8 & 6,7 & 88,0 \\
\hline Diplôme inférieur au Baccalauréat & 45,8 & 90,6 & 39,1 & 90,5 \\
\hline Baccalauréat & 18,2 & 95,5 & 19,1 & 92,5 \\
\hline Diplôme supérieur au Baccalauréat & 26,3 & 95,1 & 33,3 & 92,4 \\
\hline \multicolumn{5}{|l|}{ Caractéristiques d'emploi, 2006} \\
\hline Secteur primaire & 9,0 & 94,6 & 3,1 & 92,5 \\
\hline Secteur secondaire & 21,0 & 92,3 & 9,1 & 84,3 \\
\hline Secteur tertiaire & 70,0 & 92,5 & 87,7 & 92,6 \\
\hline Privé & 66,7 & 91,8 & 58,9 & 90,8 \\
\hline Public & 19,1 & 93,5 & 29,1 & 92,8 \\
\hline Indépendant & 10,9 & 97,0 & 6,6 & 95,8 \\
\hline Agriculteurs exploitants & 4,7 & 98,9 & 1,2 & 94,0 \\
\hline Artisans, commerçants, chefs d'entreprise & 7,0 & 96,4 & 4,3 & 94,9 \\
\hline Cadres et professions intellectuelles sup. & 16,4 & 94,9 & 11,1 & 92,3 \\
\hline Professions Intermédiaires & 24,1 & 92,8 & 22,2 & 91,7 \\
\hline Employés & 12,7 & 93,8 & 45,1 & 92,5 \\
\hline Ouvriers & 29,8 & 89,5 & 9,2 & 85,8 \\
\hline Travail à temps partiel & 3,0 & 87,5 & 30,7 & 90,3 \\
\hline Travail à temps plein & 97,0 & 92,9 & 69,3 & 92,4 \\
\hline \multicolumn{5}{|l|}{ Santé générale, 2006} \\
\hline Bonne santé perçue & 82,1 & 93,8 & 77,8 & 92,9 \\
\hline Mauvaise santé perçue & 17,9 & 86,2 & 22,2 & 85,6 \\
\hline Pas de maladie chronique & 75,3 & 93,6 & 71,9 & 91,8 \\
\hline Maladie chronique & 24,7 & 88,8 & 28,1 & 90,0 \\
\hline Pas de limitation d'activités & 90,7 & 93,4 & 88,5 & 92,1 \\
\hline Est limité dans ses activités & 9,3 & 83,2 & 11,5 & $85,4 \rightarrow$ \\
\hline
\end{tabular}




\begin{tabular}{|c|c|c|c|c|}
\hline & \multicolumn{2}{|c|}{ Hommes ( \%) } & \multicolumn{2}{|c|}{ Femmes ( \%) } \\
\hline & Proportion & $\begin{array}{c}\text { Probabilité } \\
\text { d'être en emploi } \\
\text { en } 2010\end{array}$ & Proportion & $\begin{array}{c}\text { Probabilité } \\
\text { d'être en emploi } \\
\text { en } 2010\end{array}$ \\
\hline \multicolumn{5}{|l|}{ Comportements à risque, 2006} \\
\hline Fumeur quotidien & 27,5 & 90,4 & 23,6 & 90,8 \\
\hline Pas fumeur quotidien & 72,5 & 93,2 & 76,4 & 91,8 \\
\hline Buveur à risque & 46,2 & 92,5 & 13,6 & 89,9 \\
\hline Pas buveur à risque & 53,8 & 92,4 & 86,4 & 91,6 \\
\hline Surcharge pondérale & 51,3 & 92,7 & 28,5 & 88,8 \\
\hline Poids normal ou sous poids & 48,7 & 92,5 & 71,5 & 92,4 \\
\hline \multicolumn{5}{|l|}{ Itinéraire professionnel passé } \\
\hline Majorité du parcours en emploi long & 83,5 & 92,6 & 71,7 & 92,4 \\
\hline Majorité du parcours hors d'emploi long & 16,5 & 92,0 & 28,3 & 88,5 \\
\hline Itinéraire professionnel stable & 74,3 & 92,9 & 68,9 & 92,2 \\
\hline Itinéraire professionnel instable & 25,7 & 91,2 & 31,1 & 89,4 \\
\hline
\end{tabular}

Lecture : statistiques pondérées et calées. 82 \% des hommes souffrant d'au moins un trouble mental en 2006 sont en emploi en 2010 contre 93,1\% chez ceux n'en souffrant pas.

Champ : personnes en emploi et âgées de 30 à 55 ans en 2006.

Source : enquête Santé et itinéraire professionnel, Insee.

Tableau $\mathrm{F}$

Statut en emploi en 2006, selon l'état de santé mentale

\begin{tabular}{|l|c|c|c|c|}
\cline { 2 - 5 } \multicolumn{1}{c|}{} & \multicolumn{2}{c|}{ Hommes } & \multicolumn{2}{c|}{ Femmes } \\
\cline { 2 - 5 } \multicolumn{1}{c|}{} & En emploi & Pas en emploi & En emploi & Pas en emploi \\
\hline Santé mentale en 2006 & 68,6 & 31,4 & 64,5 & 35,5 \\
A au moins un trouble mental & 90,9 & 9,1 & 77,0 & 23,0 \\
Pas de trouble mental & \multicolumn{3}{|c|}{} \\
\hline
\end{tabular}

Lecture: statistiques pondérées et calées. $68,6 \%$ des hommes souffrant d'au moins un trouble mental en 2006 sont en emploi à la même date, contre $64,5 \%$ des femmes dans la même situation.

Champ : personnes âgées de 30 à 55 ans en 2006.

Source : enquête Santé et itinéraire professionnel, Insee.

Tableau G

État de santé mentale en 2010 des personnes en emploi en 2006 ayant déclaré un trouble de santé mentale à la même date

\begin{tabular}{|l|c|c|c|c|}
\multicolumn{1}{c|}{ Hommes } & \multicolumn{2}{c|}{ Femmes } \\
\cline { 2 - 5 } \multicolumn{1}{c|}{} & $\begin{array}{c}\text { Au moins un trouble } \\
\text { mental en } 2010\end{array}$ & $\begin{array}{c}\text { Pas de trouble mental } \\
\text { en } 2010\end{array}$ & $\begin{array}{c}\text { Au moins un trouble } \\
\text { mental en } 2010\end{array}$ & $\begin{array}{c}\text { Pas de trouble mental } \\
\text { en } 2010\end{array}$ \\
\cline { 2 - 5 } Santé mentale en 2006 & 67,9 & 32,1 & 69,6 & 30,4 \\
A au moins un trouble mental & 15,7 & 84,3 & 29,8 & 70,2 \\
- dont EDC & 59,4 & 40,6 & 56,6 & 43,4 \\
\hline
\end{tabular}

Lecture : statistiques pondérées et calées. $67,9 \%$ des hommes souffrant d'au moins un trouble mental en 2006 et en emploi à la même date souffrent toujours d'un trouble mental en 2010, contre 69,6 \% des femmes dans la même situation.

Champ : personnes âgées de 30 à 55 ans en 2006, en emploi et souffrant d'au moins un trouble mental à la même date.

Source : enquête Santé et itinéraire professionnel, Insee. 
ANNEXE 4

\section{VALIDATION DES INSTRUMENTS}

Tableau A

Corrélations des variables identifiantes (population masculine)

\begin{tabular}{|l|c|c|c|}
\cline { 2 - 4 } \multicolumn{1}{c|}{} & \multicolumn{2}{c|}{ Coefficients de corrélation } & \multirow{2}{*}{ Effectifs } \\
\hline Violences subies durant l'enfance & Emploi 2010 & Santé mentale 2006 & 2004 \\
Nombreuses ruptures conjugales & $-0,04$ & $0,07^{\star \star}$ & 2004 \\
\hline
\end{tabular}

Lecture : ${ }^{* * *}$ : significatif au seuil de $1 \% ;{ }^{* *}$ : significatif au seuil de $5 \% ;{ }^{*}$ : significatif au seuil de $10 \%$.

Champ : hommes en emploi et âgés de 30 à 55 ans en 2006.

Source : enquête Santé et itinéraire professionnel, Insee.

Tableau B

Corrélations des variables identifiantes (population féminine)

\begin{tabular}{|l|c|c|c|}
\cline { 2 - 4 } \multicolumn{1}{c|}{} & \multicolumn{2}{c|}{ Coefficients de corrélation } & \multirow{2}{*}{ Effectifs } \\
\hline Violences subies durant l'enfance & Emploi 2010 & Santé mentale 2006 & 2129 \\
Élevé par un seul parent & $-0,01$ & $0,09^{\star \star \star}$ & 2129 \\
\hline
\end{tabular}

Lecture : *** : significatif au seuil de $1 \%$; ** : significatif au seuil de $5 \%$; * : significatif au seuil de $10 \%$.

Champ : femmes en emploi et âgées de 30 à 55 ans en 2006.

Source : enquête Santé et itinéraire professionnel, Insee. 
Tableau C

Estimation de la santé mentale en 2006

\begin{tabular}{|c|c|c|c|c|}
\hline & Uniprobit (Hommes) & Biprobit (Hommes) & Uniprobit (Femmes) & Biprobit (Femmes) \\
\hline Constante & $-2,50^{\star \star \star}$ & $-2,42^{\star \star \star}$ & $-2,26^{\star \star \star}$ & $-2,27^{\star \star \star}$ \\
\hline \multicolumn{5}{|l|}{ Variables identifiantes (hommes) } \\
\hline Violences durant l'enfance & $0,70^{*}$ & $0,92^{*}$ & & \\
\hline Ruptures conjugales & $0,16^{\star}$ & $0,23^{\star \star}$ & & \\
\hline \multicolumn{5}{|l|}{ Variables identifiantes (femmes) } \\
\hline Violences durant l'enfance & & & $0,48^{\star \star \star}$ & $0,47^{\star \star \star}$ \\
\hline Élevée par un seul parent & & & $0,39^{\star \star \star}$ & $0,41^{\star \star \star}$ \\
\hline \multicolumn{5}{|l|}{ Caractéristiques individuelles 2006} \\
\hline \multicolumn{5}{|l|}{ Âge (réf. : 30-35 ans) } \\
\hline $35-39$ ans & $0,48^{\star \star}$ & $0,50^{\star *}$ & $-0,15$ & $-0,16$ \\
\hline $40-44$ ans & 0,10 & 0,06 & 0,11 & 0,11 \\
\hline $45-49$ ans & 0,20 & 0,16 & 0,01 & 0,02 \\
\hline $50-55$ ans & 0,22 & 0,20 & 0,08 & 0,07 \\
\hline En couple (réf. : Seul) & $-0,45^{\star \star \star}$ & $-0,47^{\star \star \star}$ & $-0,17^{\star \star}$ & $-0,17^{\star \star}$ \\
\hline Présence d'enfants (réf : Pas d'enfant) & 0,24 & 0,26 & 0,08 & 0,09 \\
\hline \multicolumn{5}{|l|}{ Niveau de diplôme (réf. : Bac.) } \\
\hline Pas de diplôme & $-0,19$ & $-0,21$ & $-0,18$ & $-0,15$ \\
\hline Niveau inférieur au Bac & $-0,01$ & $-0,01$ & 0,05 & 0,04 \\
\hline Niveau supérieur au Bac & $-0,01$ & $-0,08$ & 0,02 & 0,01 \\
\hline \multicolumn{5}{|l|}{ Emploi en 2006} \\
\hline \multicolumn{5}{|l|}{ Sect. d'activité (réf. : Secondaire) } \\
\hline Secteur primaire & 0,11 & 0,13 & $-0,14$ & $-0,14$ \\
\hline Secteur tertiaire & 0,18 & 0,17 & $-0,15$ & $-0,15$ \\
\hline \multicolumn{5}{|l|}{ Statut d'activité (réf. : Privé) } \\
\hline Public & $-0,02$ & $-0,06$ & $-0,20^{\star \star}$ & $-0,19^{\star \star}$ \\
\hline Indépendant & $0,46^{\star}$ & $0,40^{*}$ & $-0,22$ & $-0,21$ \\
\hline \multicolumn{5}{|l|}{ PCS (réf. : Ouvrier) } \\
\hline Agriculteurs exploitants & $-0,82^{*}$ & $-0,80^{\star}$ & 0,28 & 0,27 \\
\hline Artisans, ch. d'Entr. & -019 & $-0,16$ & 0,40 & 0,39 \\
\hline Cadres et prof. Intel. Sup. & 0,24 & 0,23 & 0,03 & 0,01 \\
\hline Professions Intermédiaires & $-0,02$ & $-0,05$ & $-0,04$ & $-0,04$ \\
\hline Employés & $-0,25$ & $-0,29$ & 0,05 & 0,04 \\
\hline Temps partiel (réf. : Temps plein) & $-0,26$ & $-0,26$ & 0,14 & 0,13 \\
\hline \multicolumn{5}{|l|}{ État de santé générale en 2006} \\
\hline Mauvais état de santé perçu & $0,92^{\star \star \star}$ & $0,92^{\star \star \star}$ & $0,77^{\star \star \star}$ & $0,77^{\star \star \star}$ \\
\hline Malade chronique & 0,01 & 0,04 & 0,13 & 0,12 \\
\hline Limité dans les activités quotidiennes & 0,14 & 0,14 & $0,20^{*}$ & 0,17 \\
\hline \multicolumn{5}{|l|}{ Comportements à risque en 2006} \\
\hline Fumeur quotidien & 0,05 & 0,06 & 0,14 & 0,14 \\
\hline Buveur à risque & 0,06 & 0,08 & 0,17 & 0,16 \\
\hline Surcharge pondérale & $-0,13$ & $-0,12$ & $-0,13$ & $-0,12$ \\
\hline \multicolumn{5}{|l|}{ Itinéraire professionnel passé } \\
\hline Majorité du parcours en emploi long & $-0,01$ & 0,01 & $-0,03$ & $-0,02$ \\
\hline Itinéraire professionnel stable & $-0,13$ & $-0,15$ & 0,06 & 0,07 \\
\hline $\mathbf{N}$ & 1876 & 1860 & 2143 & 1982 \\
\hline
\end{tabular}

Lecture : ${ }^{* *}$ : significatif au seuil de $1 \% ;{ }^{* *}$ : significatif au seuil de $5 \% ;{ }^{*}$ : significatif au seuil de $10 \%$.

Champ : personnes en emploi et âgées de 30 à 55 ans en 2006.

Source : enquête Santé et itinéraire professionnel, Insee. 